\title{
On limit theory for Lévy semi-stationary
}

\section{processes}

\author{
ANDREAS BASSE-O'CONNOR*, CLAUDIO HEINRICH ${ }^{* *}$ and \\ MARK PODOLSKIJ ${ }^{\dagger}$ \\ Department of Mathematics, Aarhus University, Ny Munkegade 118, 8000 Aarhus C, Denmark. \\ E-mail: ${ }^{*}$ basse@math.au.dk; ${ }^{* *}$ claudio.heinrich@math.au.dk; ${ }^{\dagger}$ mpodolskij@math.au.dk
}

In this paper, we present some limit theorems for power variation of Lévy semi-stationary processes in the setting of infill asymptotics. Lévy semi-stationary processes, which are a one-dimensional analogue of ambit fields, are moving average type processes with a multiplicative random component, which is usually referred to as volatility or intermittency. From the mathematical point of view this work extends the asymptotic theory investigated in (Power variation for a class of stationary increments Lévy driven moving averages. Preprint), where the authors derived the limit theory for $k$ th order increments of stationary increments Lévy driven moving averages. The asymptotic results turn out to heavily depend on the interplay between the given order of the increments, the considered power $p>0$, the Blumenthal-Getoor index $\beta \in(0,2)$ of the driving pure jump Lévy process $L$ and the behaviour of the kernel function $g$ at 0 determined by the power $\alpha$. In this paper, we will study the first order asymptotic theory for Lévy semi-stationary processes with a random volatility/intermittency component and present some statistical applications of the probabilistic results.

Keywords: high frequency data; Lévy semi-stationary processes; limit theorems; power variation; stable convergence

\section{Introduction and main results}

Over the last ten years there has been a growing interest in the theory of ambit fields. Ambit fields is a class of spatio-temporal stochastic processes that has been originally introduced by Barndorff-Nielsen and Schmiegel in a series of papers [10-12] in the context of turbulence modelling, but which has found manifold applications in mathematical finance and biology among other sciences; see, for example, [2,8].

Ambit processes describe the dynamics in a stochastically developing field, for instance, a turbulent wind field, along curves embedded in such a field. A key characteristic of the modelling framework is that beyond the most basic kind of random noise it also specifically incorporates additional, often drastically changing, inputs referred to as volatility or intermittency. In terms of mathematical formulae, an ambit field is specified via

$$
X_{t}(x)=\mu+\int_{A_{t}(x)} g(t, s, x, \xi) \sigma_{s}(\xi) L(d s, d \xi)+\int_{D_{t}(x)} q(t, s, x, \xi) a_{s}(\xi) d s d \xi,
$$

where $t$ denotes time while $x$ gives the position in space. Further, $A_{t}(x)$ and $D_{t}(x)$ are Borel measurable subsets of $\mathbb{R} \times \mathbb{R}^{d}, g$ and $q$ are deterministic weight functions, $\sigma$ represents the 
intermittency field, $a$ is a drift field and $L$ denotes an independently scattered infinitely divisible random measure on $\mathbb{R} \times \mathbb{R}^{d}$ (see, e.g., [30] for details). In the literature, the sets $A_{t}(x)$ and $D_{t}(x)$ are usually referred to as ambit sets. In the framework of turbulence modelling, the stochastic field $\left(X_{t}(x)\right)_{t \geq 0, x \in \mathbb{R}^{3}}$ describes the velocity of a turbulent flow at time $t$ and position $x$, while the ambit sets $A_{t}(x), D_{t}(x)$ are typically bounded.

In this paper, we consider a purely temporal analogue of ambit fields (without drift) $\left(X_{t}\right)_{t \in \mathbb{R}}$, defined on a filtered probability space $\left(\Omega, \mathcal{F},\left(\mathcal{F}_{t}\right)_{t \in \mathbb{R}}, \mathbb{P}\right)$, which is given as

$$
X_{t}=\int_{-\infty}^{t}\left\{g(t-s)-g_{0}(-s)\right\} \sigma_{s-} d L_{s},
$$

and is usually referred to as a Lévy semi-stationary $(L S S)$ process. Here $L=\left(L_{t}\right)_{t \in \mathbb{R}}$ is a symmetric Lévy process on $\mathbb{R}$ with respect to $\left(\mathcal{F}_{t}\right)_{t \in \mathbb{R}}$ with $L_{0}=0$ and without a Gaussian component. That is, for all $u \in \mathbb{R}$, the process $\left(L_{t+u}-L_{u}\right)_{t \geq 0}$ is a symmetric Lévy process on $\mathbb{R}_{+}$with respect to $\left(\mathcal{F}_{t+u}\right)_{t \geq 0}$. The process $\left(\sigma_{t}\right)_{t \in \mathbb{R}}$ is assumed to be càdlàg and adapted to $\left(\mathcal{F}_{t}\right)_{t \in \mathbb{R}}$, and $g$ and $g_{0}$ are deterministic continuous functions vanishing on $(-\infty, 0)$. The name Lévy semi-stationary process refers to the fact that the process $\left(X_{t}\right)_{t \in \mathbb{R}}$ is stationary whenever $g_{0}=0$ and $\left(\sigma_{t}\right)_{t \in \mathbb{R}}$ is stationary and independent of $\left(L_{t}\right)_{t \in \mathbb{R}}$. It is assumed throughout this paper that $g, g_{0}, \sigma$ and $L$ are such that the process $\left(X_{t}\right)$ is well-defined, which is in particular satisfied under the conditions stated in Remark 3.3 below. We are interested in the asymptotic behaviour of the power variation of the process $X$. More precisely, let us consider the $k$ th order increments $\Delta_{i, k}^{n} X$ of $X, k \in \mathbb{N}$, that are defined by

$$
\Delta_{i, k}^{n} X:=\sum_{j=0}^{k}(-1)^{j}\left(\begin{array}{l}
k \\
j
\end{array}\right) X_{(i-j) / n}, \quad \text { where } i \geq k
$$

For instance, we have that $\Delta_{i, 1}^{n} X=X_{\frac{i}{n}}-X_{\frac{i-1}{n}}$ and $\Delta_{i, 2}^{n} X=X_{\frac{i}{n}}-2 X_{\frac{i-1}{n}}+X_{\frac{i-2}{n}}$. The main functional of interest is the power variation computed on the basis of $k$ th order increments:

$$
V(p ; k)_{t}^{n}:=\sum_{i=k}^{[n t]}\left|\Delta_{i, k}^{n} X\right|^{p}, \quad p>0 .
$$

At this stage, we remark that power variation of stochastic processes has been a very active research area in the last decade. We refer for example, to [7,23,24,29] for limit theory for power variations of Itô semimartingales, to $[3,6,18,22,28]$ for the asymptotic results in the framework of fractional Brownian motion and related processes, and to [17,34] for investigations of power variation of the Rosenblatt process. The power variation of Brownian semi-stationary processes, which is the model (1.2) driven by a Brownian motion, has been studied in [4,5,20]. Under proper normalisation, the authors have shown convergence in probability for the statistic $V(p ; k)_{t}^{n}$ and proved its asymptotic mixed normality.

However, when the driving motion in (1.2) is a pure jump Lévy process, the asymptotic theory is very different from the Brownian case. In the recent work [13], the power variation of the model (1.2) with constant intermittency $\sigma$ has been studied. The authors showed that the asymptotic 
behavior of $V(p ; k)_{t}^{n}$ is greatly affected by the Blumenthal-Getoor index $\beta$ of the driving Lévy motion as well as the behavior of the kernel function $g$ at 0 . The goal of this work is to extend the result of [13] to LSS-processes with nontrivial intermittency process $\sigma$. Such extensions are important in applications, say in the framework of turbulence, since the intermittency is often the main object of interest. Moreover, we show that the convergence holds functional with respect to the Skorokhod $M_{1}$-topology in the setting of Theorem 1.1(i), and with respect to the uniform norm in the settings of Theorem 1.1(ii) and (iii).

Throughout this article, $\beta$ denotes the Blumenthal-Getoor index of the driving Lévy process, which is defined as

$$
\beta:=\inf \left\{r \geq 0: \int_{-1}^{1}|x|^{r} v(d x)<\infty\right\} \in[0,2],
$$

where $v$ denotes the Lévy measure of $L$. It is well known that $\sum_{s \in[0,1]}\left|\Delta L_{s}\right|^{p}$ is finite when $p>$ $\beta$, while it is infinite for $p<\beta$. Here $\Delta L_{s}=L_{s}-L_{s-}$ where $L_{s-}=\lim _{u \uparrow s, u<s} L_{u}$. We recall that for a stable Lévy processes the Blumenthal-Getoor index matches the index of stability. The authors of [13] impose the following set of assumptions on $g, g_{0}$ and $v$, which we assume to hold throughout this paper.

Assumption (A). The function $g: \mathbb{R} \rightarrow \mathbb{R}$ satisfies $\lim _{t \downarrow 0} g(t) t^{-\alpha}=c_{0}$ for some $\alpha>0$ and $c_{0} \neq 0$. There is a $\theta \in(0,2]$, such that $\limsup _{t \rightarrow \infty} v(x:|x| \geq t) t^{\theta}<\infty$ and $g-g_{0}$ is a bounded function in $L^{\theta}\left(\mathbb{R}_{+}\right)$. Furthermore, $g$ is $k$-times continuously differentiable on $(0, \infty)$ and there exists a $\delta>0$ such that $\left|g^{(k)}(t)\right| \leq C t^{\alpha-k}$ for all $t \in(0, \delta)$, and such that both $\left|g^{\prime}\right|$ and $\left|g^{(k)}\right|$ are in $L^{\theta}((\delta, \infty))$ and are decreasing on $(\delta, \infty)$.

Assumption (A-log). In addition to (A), suppose that $\int_{\delta}^{\infty}\left|g^{(k)}(s)\right|^{\theta} \log \left(1 /\left|g^{(k)}(s)\right|\right) d s<\infty$.

Assumption (A) ensures, in particular, that the process $X$ with $\sigma=1$ is well-defined, cf. [13]. When $L$ is a $\beta$-stable Lévy process, we can and will always choose $\theta=\beta$ in Assumption (A). In addition to these assumptions, we use in our main result the following integrability conditions on the stochastic process $H_{s}:=g^{(k)}(-s) \sigma_{s} \mathbb{1}_{(-\infty,-\delta]}(s), s \in \mathbb{R}$, where $\delta$ is defined as in Assumption (A).

Assumption (B1). There exists $\rho>0$ with $\rho \leq 1 \wedge \theta$ and $\beta^{\prime}>\beta$ with $\beta^{\prime} \geq p$ such that

$$
\mathbb{E}\left[\left(\int_{\mathbb{R}}\left(\left|H_{s}\right|^{\rho} \vee\left|H_{s}\right|^{\beta^{\prime}}\right) d s\right)^{1 \vee \frac{p}{2}}\right]<\infty .
$$

For $\theta=1$ suppose in addition that we may choose $\rho<1$ in (1.4).

Assumption (B2). It holds that

$$
\mathbb{E}\left[\int_{\mathbb{R}}\left|H_{s}\right|^{\beta} d s\right]<\infty .
$$

For $p \leq 2$ it is not difficult to show that (B1) is at least satisfied when we can choose $\theta<1$ in (A), and the intermittency satisfies $\sup _{s \in(-\infty,-\delta]} \mathbb{E}\left[\left|\sigma_{s}\right|^{1 \vee \beta^{\prime}}\right]<\infty$. Assumption (B2) will only 
be used in the case where $L$ is a $\beta$-stable Lévy motion (see Theorem 1.1(ii) below), and is e.g. satisfied when $\sup _{s \in(-\infty,-\delta]} \mathbb{E}\left[\left|\sigma_{s}\right|^{\beta}\right]<\infty$. These stronger assumptions are satisfied in many applications, as $\sigma$ is often assumed to be stationary.

Before we state our main theorem, we introduce some more notation. Let $h_{k}: \mathbb{R} \rightarrow \mathbb{R}$ be given by

$$
h_{k}(x)=\sum_{j=0}^{k}(-1)^{j}\left(\begin{array}{l}
k \\
j
\end{array}\right)(x-j)_{+}^{\alpha}, \quad x \in \mathbb{R},
$$

where $y_{+}=\max \{y, 0\}$ for all $y \in \mathbb{R}$. Let $\mathbb{F}=\left(\mathcal{F}_{t}\right)_{t \geq 0}$ and $\left(T_{m}\right)_{m \geq 1}$ be a sequence of $\mathbb{F}$-stopping times that exhausts the jumps of $\left(L_{t}\right)_{t \geq 0}$. That is, $\left\{T_{m}(\omega): m \geq 1\right\} \cap[0, \infty)=\left\{t \geq 0: \Delta L_{t}(\omega) \neq\right.$ $0\}$ and $T_{m}(\omega) \neq T_{n}(\omega)$ for all $m \neq n$ with $T_{m}(\omega)<\infty$. Let $\left(U_{m}\right)_{m \geq 1}$ be independent and uniform $[0,1]$-distributed random variables, defined on an extension $\left(\Omega^{\prime}, \overline{\mathcal{F}}^{\prime}, \mathbb{P}^{\prime}\right)$ of the original probability space, which are independent of $\mathcal{F}$. By $\left(\mathbb{D}\left(\mathbb{R}_{+} ; \mathbb{R}\right), M_{1}\right)$, we denote the Skorokhod space of càdlàg functions from $\mathbb{R}_{+}$into $\mathbb{R}$, equipped with the Skorokhod $M_{1}$-topology, making it a Polish space. The $M_{1}$-topology was originally introduced in [33]. We give a definition in Section 4, a detailed account and many properties can be found in [35]. For stochastic processes $Z^{n}, Z$ with càdlàg sample paths that are defined on $\left(\Omega^{\prime}, \mathcal{F}^{\prime}\right)$, we denote by $Z^{n} \stackrel{\mathcal{L}_{M_{1}}-s}{\longrightarrow} Z$ the functional $\mathcal{F}$-stable convergence in law with respect to the $M_{1}$-topology. That is, $Z^{n} \stackrel{\mathcal{L}_{M_{1}}-s}{\longrightarrow} Z$ means that $\mathbb{E}^{\prime}\left[\phi\left(Z^{n}\right) Y\right] \rightarrow \mathbb{E}^{\prime}[\phi(Z) Y]$ for all bounded continuous functions $\phi:\left(\mathbb{D}\left(\mathbb{R}_{+} ; \mathbb{R}\right), M_{1}\right) \rightarrow \mathbb{R}$, and all bounded $\mathcal{F}$-measurable $Y$, where $\mathbb{E}^{\prime}$ denotes the expectation on the extended space $\left(\Omega^{\prime}, \mathcal{F}^{\prime}, \mathbb{P}^{\prime}\right)$. By $\stackrel{\text { u.c.p. }}{\longrightarrow}$ we denote uniform convergence on compact sets in probability. That is, $\left(Z_{t}^{n}\right)_{t \geq 0} \stackrel{\text { u.c.p. }}{\longrightarrow}\left(Z_{t}\right)_{t \geq 0}$ as $n \rightarrow \infty$ means that $\mathbb{P}\left(\sup _{t \in[0, N]}\left|Z_{t}^{n}-Z_{t}\right|>\varepsilon\right) \rightarrow 0$ for all $N \in \mathbb{N}$ and all $\varepsilon>0$.

The following extension of [13], Theorem 1.1, to include a non-trivial $\sigma$ process and functional convergence, is the main result of this paper.

Theorem 1.1. Let $X=\left(X_{t}\right)_{t \geq 0}$ be a stochastic process defined by (1.2). Let (A) be satisfied and assume that the Blumenthal-Getoor index satisfies $\beta<2$.

(i) Suppose that (B1) holds and if $\theta=1$ assume additionally that (A-log) is satisfied. Let $\alpha<k-1 / p, p>\beta$ and $p \geq 1$. Then, as $n \rightarrow \infty$, the functional $\mathcal{F}$-stable convergence holds

$$
n^{\alpha p} V(p ; k)_{t}^{n} \stackrel{\mathcal{L}_{M_{1}-s}}{\longrightarrow}\left|c_{0}\right|^{p} \sum_{m: T_{m} \in[0, t]}\left|\Delta L_{T_{m}} \sigma_{T_{m}-}\right|^{p} V_{m} \quad \text { where } V_{m}=\sum_{l=0}^{\infty}\left|h_{k}\left(l+U_{m}\right)\right|^{p}
$$

(ii) Suppose that $L$ is a symmetric $\beta$-stable Lévy process with $\beta \in(0,2)$ and scale parameter $\gamma>0$. Suppose that (B2) holds and that $\alpha<k-1 / \beta$ and $p<\beta$. Then as $n \rightarrow \infty$

$$
n^{-1+p(\alpha+1 / \beta)} V(p ; k)_{t}^{n} \stackrel{\text { u.c.p. }}{\longrightarrow} m_{p} \int_{0}^{t}\left|\sigma_{s}\right|^{p} d s,
$$


where $m_{p}=\left|c_{0}\right|^{p} \gamma^{p}\left(\int_{\mathbb{R}}\left|h_{k}(x)\right|^{\beta} d x\right)^{p / \beta} \mathbb{E}\left[|Z|^{p}\right]$, where $Z$ is a symmetric $\beta$-stable random variable with scale parameter 1 .

(iii) Suppose that (B1) holds, $\theta>1, \alpha>k-1 /(\beta \vee p)$ and $p \geq 1$. If $p=\theta$ assume additionally that (A-log) is satisfied. Then, as $n \rightarrow \infty$,

$$
n^{-1+p k} V(p ; k)_{t}^{n} \stackrel{\text { u.c.p. }}{\longrightarrow} \int_{0}^{t}\left|F_{u}\right|^{p} d u,
$$

where $\left(F_{u}\right)_{u \in \mathbb{R}}$ is a version with measurable sample paths of the process defined by

$$
F_{u}=\int_{-\infty}^{u} g^{(k)}(u-s) \sigma_{s-} d L_{s} \quad \text { a.s. for all } u \in \mathbb{R},
$$

which necessarily satisfies $\int_{0}^{t}\left|F_{u}\right|^{p} d u<\infty$, almost surely.

Under the integrability assumptions (B1) and (B2), Theorem 1.1 covers all possible choices of $\alpha>0, \beta \in[0,2)$ and $p \geq 1$ except the critical cases where $p=\beta, \alpha=k-1 / p$ or $\alpha=k-1 / \beta$. The two critical cases $\alpha=k-1 / p, p>\beta$ and $\alpha=k-1 / \beta, p<\beta$ have been studied in [14] in the case $\sigma \equiv 1$. We conjecture that analogous results hold for LSS processes with non-trivial intermittency component, but will not pursue this theory in the paper.

First order asymptotic theory for Lévy semi-stationary processes can be used to draw inference on the parameters $\alpha, \beta$ and on certain intermittency functionals in the context of high frequency observations, see Section 2. Furthermore, this type of limit theory is an intermediate step towards asymptotic results for general ambit fields of the form (1.1). We remark that, in contrast to the Brownian setting, extending the first order limit theory presented in [13] to Lévy semi-stationary processes with non-trivial $\sigma$ is a more complex issue. This is due to the fact that it is harder to estimate various norms of $X$ and related processes when the driving process $L$ is a Lévy process. To this end, we rely heavily on decoupling techniques and isometries for stochastic integral mappings presented in the monograph [26] and [31], see Section 3 for more details.

This paper is structured as follows. Section 2 is devoted to various statistical applications of our limit theory. In Section 3, we discuss properties of Lévy integrals of predictable processes and recall essential estimates from [26] for those integrals. All proofs are demonstrated in Section 4.

\section{Some statistical applications}

We start this section by giving an interpretation to the parameters $\alpha>0$ and $\beta \in(0,2)$. Let us consider the linear fractional stable motion defined by

$$
Y_{t}:=c_{0} \int_{\mathbb{R}}\left\{(t-s)_{+}^{\alpha}-(-s)_{+}^{\alpha}\right\} d L_{s},
$$

where $L$ is symmetric $\beta$-stable, and the constant $c_{0}$ has been introduced in Assumption (A). It is well known that the process $\left(Y_{t}\right)_{t \geq 0}$ is well defined whenever $H=\alpha+1 / \beta<1$. Furthermore, 
the process $\left(Y_{t}\right)_{t \geq 0}$ has stationary symmetric $\beta$-stable increments, Hölder continuous paths of all orders smaller than $\alpha$ and self-similarity index $H$, that is,

$$
\left(Y_{a t}\right)_{t \geq 0} \stackrel{d}{=}\left(a^{H} Y_{t}\right)_{t \geq 0} \quad \text { for any } a \in \mathbb{R}_{+} .
$$

We refer to for example, [15] for more details. As it has been discussed in [13,14] in the setting $\sigma=1$, the small scale behaviour of the process $X$ is well approximated by the corresponding behaviour of the linear fractional stable motion $Y$. In other words, when the intermittency process $\sigma$ is smooth, we have that

$$
X_{t+\Delta}-X_{t} \approx \sigma_{t}\left(Y_{t+\Delta}-Y_{t}\right)
$$

for small $\Delta>0$. Thus, intuitively speaking, the properties of $Y$ (Hölder smoothness, selfsimilarity) transfer to the process $X$ on small scales.

Having understood the role of the parameters $\alpha>0$ and $H=\alpha+1 / \beta \in(1 / 2,1)$ from the modelling perspective, it is obviously important to investigate estimation methods for these parameters. We note that the conditions $\alpha>0$ and $H \in(1 / 2,1)$ imply the restrictions $\beta \in(1,2)$ and $\alpha<1-1 / \max \{p, \beta\}$. Hence, the regime of Theorem 1.1(iii) is never applicable.

We start with a direct estimation procedure, which identifies the convergence rates in Theorem 1.1(i)-(ii). We apply these convergence results only for $t=1$ and $k=1$. For $p \in[\underline{p}, \bar{p}]$ with $\underline{p} \in(0,1)$ and $\bar{p}>2$, we introduce the statistic

$$
S(n, p):=-\frac{\log V(p)^{n}}{\log n} \quad \text { with } V(p)^{n}=V(p ; 1)_{1}^{n} .
$$

When the underlying Lévy motion $L$ is symmetric $\beta$-stable and the assumptions of Theorems 1.1(i)-(ii) are satisfied, we obtain that

$$
S(n, p) \stackrel{\mathbb{P}}{\longrightarrow} S_{\alpha, \beta}(p):= \begin{cases}\alpha p: & \alpha<1-1 / p \text { and } p>\beta, \\ p H-1: & \alpha<1-1 / \beta \text { and } p<\beta,\end{cases}
$$

if the parameter is $(\alpha, \beta)$. Indeed, the result of Theorem 1.1(i) shows that

$$
\frac{\alpha p \log n+\log V(p)^{n}}{\log n} \stackrel{\mathcal{L}-s}{\longrightarrow} 0 \Rightarrow \frac{\alpha p \log n+\log V(p)^{n}}{\log n} \stackrel{\mathbb{P}}{\longrightarrow} 0 .
$$

This explains the first line in (2.1), and the second line follows similarly from Theorem 1.1(ii). At this stage we remark that the limit $S_{\alpha, \beta}:[\underline{p}, \bar{p}] \backslash\{\beta\} \rightarrow \mathbb{R}$ is a piecewise linear function with two different slopes. It can be continuously extended to the function $S_{\alpha, \beta}:[\underline{p}, \bar{p}] \rightarrow \mathbb{R}$, whose definition can be further extended to include all values

$$
(\alpha, \beta) \in J:=\left\{(\alpha, \beta) \in \mathbb{R}^{2}: \beta \in[1,2], \alpha \in[0,1-1 / \beta]\right\} .
$$

For estimation of $(\alpha, \beta)$, it is natural to minimise the $L^{2}$-distance between the observed scale function $S(n, p)$ and the theoretical limit $S_{\alpha, \beta}(p)$ :

$$
\left(\hat{\alpha}_{n}, \hat{\beta}_{n}\right) \in \underset{(\alpha, \beta) \in J}{\operatorname{argmin}}\left\|S(n)-S_{\alpha, \beta}\right\|_{L^{2}([\underline{p}, \bar{p}])}
$$


with $S(n):=S(n, \cdot)$. This approach is somewhat similar to the estimation method proposed in [21]. For finite $n$, the minimum of the $L^{2}([p, \bar{p}])$-distance at (2.2) is not necessarily obtained at a unique point, and we take an arbitrary measurable minimiser $\left(\hat{\alpha}_{n}, \hat{\beta}_{n}\right)$. Our next result shows consistency of the estimator $\left(\hat{\alpha}_{n}, \hat{\beta}_{n}\right)$.

Corollary 2.1. Let $\left(\alpha_{0}, \beta_{0}\right) \in J^{\circ}$, where $J^{\circ}$ is the set of all inner points of $J$, denote the true parameter of the model (1.2), and let $L$ be a symmetric $\beta_{0}$-stable Lévy motion. Assume that the conditions of Theorem 1.1(i) (resp. Theorem 1.1(ii)) hold when $\alpha_{0} \in(0,1-1 / p)$ and $p>\beta_{0}$ (resp. $\alpha_{0} \in\left(0,1-1 / \beta_{0}\right)$ and $\left.p<\beta_{0}\right)$. Then we obtain convergence in probability

$$
\left(\hat{\alpha}_{n}, \hat{\beta}_{n}\right) \stackrel{\mathbb{P}}{\longrightarrow}\left(\alpha_{0}, \beta_{0}\right) .
$$

Proof. Set $r_{0}=\left(\alpha_{0}, \beta_{0}\right)$ and $\widehat{r}_{n}=\left(\hat{\alpha}_{n}, \hat{\beta}_{n}\right)$. We first show the convergence

$$
\left\|S(n)-S_{r_{0}}\right\|_{L^{2}([\underline{p}, \bar{p}])} \stackrel{\mathbb{P}}{\longrightarrow} 0 .
$$

From (2.1), we deduce that $S(n, p) \stackrel{\mathbb{P}}{\longrightarrow} S_{r_{0}}(p)$ for all $p \in[\underline{p}, \bar{p}] \backslash\left\{\beta_{0}\right\}$. Furthermore, for any $p \in[\underline{p}, \bar{p}]$, it holds that

$$
\left(V(\bar{p})^{n}\right)^{1 / \bar{p}} \leq\left(V(p)^{n}\right)^{1 / p} \leq\left(V(\underline{p})^{n}\right)^{1 / \underline{p}} .
$$

Hence, we deduce the inequality

$$
\left|\frac{\log V(p)^{n}}{\log n}\right| \leq \max \left\{\frac{p}{\bar{p}} \cdot\left|\frac{\log V(\bar{p})^{n}}{\log n}\right|, \frac{p}{\underline{p}} \cdot\left|\frac{\log V(\underline{p})^{n}}{\log n}\right|\right\} .
$$

Since $\left|\log V(\underline{p})^{n} / \log n\right| \stackrel{\mathbb{P}}{\longrightarrow} \underline{p}\left(\alpha_{0}+1 / \beta_{0}\right)-1$ and $\left|\log V(\bar{p})^{n} / \log n\right| \stackrel{\mathbb{P}}{\longrightarrow} \alpha_{0} \bar{p}$, because $\underline{p}<$ $1<\beta_{0}$ and $\bar{p}>2>\beta_{0}$, we readily deduce the convergence at (2.3) by dominated convergence theorem.

Now, we note that the mapping $G: J \rightarrow G(J) \subset L^{2}([\underline{p}, \bar{p}]), r \mapsto S_{r}$, is a homeomorphism. Thus, it suffices to prove that $\left\|S_{\widehat{r}_{n}}-S_{r_{0}}\right\|_{L^{2}([p, \bar{p}])} \stackrel{\mathbb{P}}{\longrightarrow} 0$ to conclude $\widehat{r}_{n} \stackrel{\mathbb{P}}{\longrightarrow} r_{0}$. To show the former, we observe that

$$
\begin{aligned}
\left\|S_{\widehat{r}_{n}}-S_{r_{0}}\right\|_{L^{2}([\underline{p}, \bar{p}])} & \leq\left\|S(n)-S_{r_{0}}\right\|_{L^{2}([\underline{p}, \bar{p}])}+\left\|S(n)-S_{\widehat{r}_{n}}\right\|_{L^{2}([\underline{p}, \bar{p}])} \\
& =\left\|S(n)-S_{r_{0}}\right\|_{L^{2}([\underline{p}, \bar{p}])}+\min _{r \in J}\left\|S(n)-S_{r}\right\|_{L^{2}([\underline{p}, \bar{p}])} \\
& \leq 2\left\|S(n)-S_{r_{0}}\right\|_{L^{2}([\underline{p}, \bar{p}])} \stackrel{\mathbb{P}}{\longrightarrow} 0 .
\end{aligned}
$$

This completes the proof of Corollary 2.1.

In practice, the integral in (2.2) needs to be discretised. We further remark that the estimator $S(n, p)$ has the rate of convergence $\log n$ due to the bias $V(p) / \log n$, where $V(p)$ denotes the limit of $V(p)^{n}$. 
As for the estimation of the self-similarity parameter $H=\alpha+1 / \beta \in(1 / 2,1)$, there is an alternative estimator based on a ratio statistic. Recalling that $\beta \in(1,2)$, we deduce for any $p \in$ $(0,1]$

$$
R(n, p):=\frac{\sum_{i=2}^{n}\left|X_{\frac{i}{n}}-X_{\frac{i-2}{n}}\right|^{p}}{\sum_{i=1}^{n}\left|X_{\frac{i}{n}}-X_{\frac{i-1}{n}}\right|^{p}} \stackrel{\mathbb{P}}{\longrightarrow} 2^{p H}
$$

by a direct application of Theorem 1.1(ii). Thus, we immediately conclude that

$$
\hat{H}_{n}:=\frac{\log R(n, p)}{p \log 2} \stackrel{\mathbb{P}}{\longrightarrow} H .
$$

This type of idea is rather standard in the framework of a fractional Brownian motion with Hurst parameter $H$. It has been also applied to Brownian semi-stationary processes in [4,5]. Theorem 1.2(i) in [13], which has been shown in the setting $\sigma=1$, suggests that the statistic $\hat{H}_{n}$ has convergence rate $n^{1-1 /(1-\alpha) \beta}$ whenever $p \in(0,1 / 2]$. Furthermore, the rate of convergence can be improved to $\sqrt{n}$ via using $k$ th order increments with $k \geq 2$ (cf. [13], Theorem 1.2(ii)). However, we dispense with the precise proof of these statements for non-constant intermittency process $\sigma$. In a recent work [19], it was shown that for linear fractional stable motions the convergence $\hat{H}_{n} \stackrel{\mathbb{P}}{\longrightarrow} H$ continues to hold for powers $p \in(-1,0)$. This is particularly useful, since choosing $p$ negative ensures that the condition $p<\beta$ of Theorem 1.1(ii) is always satisfied. However, proving this result for a general Lévy semi-stationary process is a much more delicate issue.

Another important object for applications in turbulence modelling is the intermittency process $\sigma$. First of all, we remark that the process $\sigma$ in the general model (1.2) is statistically not identifiable. This is easily seen, because multiplication of $\sigma$ by a constant can not be distinguished from the multiplication of, say, Lévy process $L$ by the same constant. However, it is very well possible to estimate the relative intermittency, which is defined as

$$
\operatorname{RI}(p):=\frac{\int_{0}^{t}\left|\sigma_{s}\right|^{p} d s}{\int_{0}^{1}\left|\sigma_{s}\right|^{p} d s}, \quad t \in(0,1),
$$

for $p \in(0,1]$. The relative intermittency, which has been introduced in [9] for $p=2$ in the context of Brownian semi-stationary processes, describes the relative amplitude of the velocity process on an interval $[0,1]$. Applying the convergence result of Theorem 1.1(ii) for $p \in(0,1]$, the relative intermittency can be consistently estimated via

$$
\operatorname{RI}(n, p):=\frac{V(p)_{t}^{n}}{V(p)_{1}^{n}} \stackrel{\mathbb{P}}{\longrightarrow} \mathrm{RI}(p) .
$$

Again we suspect that the associated convergence rate is $n^{1-1 /(1-\alpha) \beta}$ whenever $p \in(0,1 / 2]$ as suggested by [13], Theorem 1.2(i). 


\section{Preliminaries: Estimates on Lévy integrals}

To prove the various limit theorems, we need very sharp estimates of the $p$ th moments of the increments of process $X$ defined in (1.2). In fact, we need such estimates for several different processes related to $X$ obtained by different truncations. When $F: \mathbb{R}_{+} \rightarrow \mathbb{R}$ is a deterministic function, the estimates for integrals $\int_{0}^{t} F_{S} d L_{s}$ go back to Rajput and Rosiński [30], Theorem 3.3. Their results imply the existence of a constant $C>0$ such that

$$
\mathbb{E}\left[\left|\int_{0}^{t} F_{S} d L_{s}\right|^{q}\right] \leq C\|F\|_{L, q}^{q},
$$

where $\|\cdot\|_{L, q}$ is a certain functional to be defined below (when $L$ is symmetric and without Gaussian component). The decoupling approach used in Kwapién and Woyczyński [26] provides an extension of the results to general predictable $F$, see Lemmas 3.1 and 3.2 below. Before stating the results precisely, we need the following notation.

Let $L=\left(L_{t}\right)_{t \in \mathbb{R}}$ be a symmetric Lévy process on the real line with $L_{0}=0$, Lévy measure $v$ and without a Gaussian component. For a predictable process $\left(F_{t}\right)_{t \in \mathbb{R}}$ and for $q=0$ or $q \geq 1$ we define

$$
\Phi_{q, L}(F):=\int_{\mathbb{R}^{2}} \phi_{q}\left(F_{s} u\right) d s v(d u), \quad \text { where } \phi_{q}(x):=|x|^{q} \mathbb{1}_{\{|x|>1\}}+x^{2} \mathbb{1}_{\{|x| \leq 1\}} .
$$

A predictable process $F=\left(F_{t}\right)_{t \in \mathbb{R}}$ is integrable with respect to $\left(L_{t}\right)_{t \in \mathbb{R}}$ in the sense of [26] if and only if $\Phi_{0, L}(F)<\infty$ almost surely (cf. [26], Theorem 9.1.1). The linear space of predictable processes satisfying $\Phi_{q, L}(F)<\infty$ will be denoted by $\mathbf{L}^{q}(d L)$. In order to estimate the $q$ th moments of stochastic integrals we introduce for all $q \geq 1$

$$
\|F\|_{q, L}:=\inf \left\{\lambda>0: \Phi_{q, L}(F / \lambda) \leq 1\right\}, \quad F \in \mathbf{L}^{q}(d L) .
$$

The following two results from [26] and [31] will play a key role for our proofs.

Lemma 3.1 ([26], equation (9.5.3)). For all $q \geq 1$ there is a constant $C$, depending only on $q$, such that we obtain for all $F \in \mathbf{L}^{q}(d L)$

$$
\mathbb{E}\left[\left|\int_{\mathbb{R}} F_{s} d L_{s}\right|^{q}\right] \leq C \mathbb{E}\left[\|F\|_{q, L}^{q}\right] .
$$

The above lemma follows by [26], equation (9.5.3), and the comments following it. Actually, [26], equation (9.5.3), only treats the case where the stochastic integral in (3.2) is over a finite time interval, say $\int_{0}^{t} F_{S} d L_{s}$. However, the definition of the stochastic integral and the estimates of the integral in [26], Chapters 8-9, extend to the case of $\int_{\mathbb{R}} F_{S} d L_{s}$ in a natural way.

For the next result, which is an immediate consequence of [31], Theorem 2.1, we use the notation $\|Z\|_{\beta, \infty}^{\beta}=\sup _{\lambda>0} \lambda^{\beta} \mathbb{P}[|Z|>\lambda]$ for an arbitrary random variable $Z$. For $q<\beta$ it holds that $\mathbb{E}\left[|Z|^{q}\right]^{1 / q} \leq\|Z\|_{\beta, \infty} \leq\left(\frac{\beta}{\beta-q}\right)^{1 / q} \mathbb{E}\left[|Z|^{\beta}\right]^{1 / \beta}$. In the literature, $\|\cdot\|_{\beta, \infty}$ is often referred to as the weak $L^{\beta}$-norm. However, $\|\cdot\|_{\beta, \infty}$ satisfies the triangle inequality only up to a constant. 
Lemma 3.2 ([31], Theorem 2.1). Let $\left(L_{t}\right)_{t \in \mathbb{R}}$ be a symmetric $\beta$-stable Lévy process. Then there is a positive constant $C>0$ such that for all $\left(F_{t}\right)_{t \in \mathbb{R}}$ in $\mathbf{L}^{0}(d L)$ it holds that

$$
\left\|\int_{\mathbb{R}} F_{S} d L_{s}\right\|_{\beta, \infty}^{\beta} \leq C \mathbb{E}\left[\int_{\mathbb{R}}\left|F_{s}\right|^{\beta} d s\right] .
$$

The next remark gives sufficient conditions for the process $X$ introduced at (1.2) to be welldefined.

Remark 3.3. Suppose that (A) is satisfied and define the two processes $F^{(1)}$ and $F^{(2)}$ by $F_{s}^{(1)}=$ $\left(g(-s)-g_{0}(-s)\right) \sigma_{s}$ and $F_{s}^{(2)}=g^{\prime}(-s) \sigma_{s}$ for $s<0$. Then the process $X$ given by (1.2) is welldefined if there exists a $\beta^{\prime}>\beta$ such that

$$
\int_{-\infty}^{-\delta}\left(\left|F_{s}^{(i)}\right|^{\theta} \mathbb{1}_{\left\{\left|F_{s}^{(i)}\right| \leq 1\right\}}+\left|F_{s}^{(i)}\right|^{\beta^{\prime}} \mathbb{1}_{\left\{\left|F_{s}^{(i)}\right|>1\right\}}\right) d s<\infty
$$

almost surely for $i=1,2$. To show the above we argue as follows: For any $\beta^{\prime} \in(\beta, 2]$ we deduce from (A) and simple calculations the estimate

$$
\int_{\mathbb{R}}\left(|u x|^{2} \wedge 1\right) v(d x) \leq C\left(|u|^{\theta} \mathbb{1}_{\{|u| \leq 1\}}+|u|^{\beta^{\prime}} \mathbb{1}_{\{|u|>1\}}\right), \quad u \in \mathbb{R} .
$$

Then, an application of the mean value theorem combined with assumption (3.3) yields that $\Phi_{0, L}\left(H^{(t)}\right)<\infty$ almost surely for all $t>0$, where $H_{s}^{(t)}=\left(g(t-s)-g_{0}(-s)\right) \sigma_{s}$. This guarantees the existence of the process $X$ due to [26], Theorem 9.1.1.

In our proofs, we will need the following properties of the functional $\|\cdot\|_{L, q}$ defined in (3.1).

(i) Homogeneity: For all $\lambda \in \mathbb{R}, F \in \mathbf{L}^{q}(d L)$, $\|\lambda F\|_{q, L}=|\lambda|\|F\|_{q, L}$.

(ii) Triangle inequality (up to a constant): There exists a constant $C>0$ such that for all $F^{1}, \ldots, F^{m} \in \mathbf{L}^{q}(d L)$ we have

$$
\left\|F^{1}+\cdots+F^{m}\right\|_{q, L} \leq C\left(\left\|F^{1}\right\|_{q, L}+\cdots+\left\|F^{m}\right\|_{q, L}\right),
$$

and the constant $C$ does not depend on $m$ or $L$.

(iii) Upper bound: For all $F \in \mathbf{L}^{q}(d L)$, we have

$$
\|F\|_{q, L} \leq \Phi_{q, L}^{1 / 2}(F) \vee \Phi_{q, L}^{1 / q}(F) .
$$

Property (i) follows directly from the definition of $\|\cdot\|_{L, q}$ in (3.1). To show property (ii) it is sufficient to derive (3.5) for $F^{1}, \ldots, F^{m} \in \mathbf{L}_{\mathrm{nr}}^{q}(d L)$, where $\mathbf{L}_{\mathrm{nr}}^{q}(d L)$ denotes the subspace of nonrandom processes in $\mathbf{L}^{q}(d L)$. We will show that there is a norm $\|\cdot\|_{q, L}^{\prime}$ on $\mathbf{L}_{\mathrm{nr}}^{q}(d L)$ and $c>0$ and $C>0$ such that $c\|F\|_{q, L}^{\prime} \leq\|F\|_{q, L} \leq C\|F\|_{q, L}^{\prime}$, for all $F \in \mathbf{L}_{\mathrm{nr}}^{q}(d L)$, which then implies (3.5). To this end, let

$$
\widetilde{\phi}_{q}(x):=\left(2 / q|x|^{q}+1-2 / q\right) \mathbb{1}_{\{|x|>1\}}+x^{2} \mathbb{1}_{\{|x| \leq 1\}} .
$$


Clearly, there exist $c, C>0$ such that $c \widetilde{\phi}_{q}(x) \leq \phi_{q}(x) \leq C \widetilde{\phi}_{q}(x)$ for all $x \in \mathbb{R}$. Since the function $\widetilde{\phi}_{q}$ is convex, the functional

$$
\|F\|_{q, L}^{\prime}=\inf \left\{\lambda \geq 0: \int_{\mathbb{R}^{2}} \widetilde{\phi}_{q}\left(F_{s} u / \lambda\right) d s v(d u) \leq 1\right\}
$$

is a norm on $\mathbf{L}_{\mathrm{nr}}^{q}(d L)$, called the Luxemburg norm (cf. [27], Chapter 1). Using convexity of $\widetilde{\phi}_{q}$ it follows by straightforward calculations that $c\|F\|_{q, L}^{\prime} \leq\|F\|_{q, L} \leq C\|F\|_{q, L}^{\prime}$ for all $F \in \mathbf{L}_{\mathrm{nr}}^{q}(d L)$. This implies (3.5). Finally, property (iii) follows by the fact that $\phi_{q}(\lambda x) \leq\left(\lambda^{2} \vee \lambda^{q}\right) \phi_{q}(x)$ for all $\lambda \geq 0$.

We conclude this subsection with a remark on the situation when the integrator is a nonsymmetric Lévy process $\left(\widetilde{L}_{t}\right)_{t \in \mathbb{R}}$ with $\widetilde{L}_{0}=0$, Lévy measure $\widetilde{v}$, shift parameter $\eta$, without a Gaussian part, and the truncation function $\tau: x \mapsto \mathbb{1}_{\{|x|<1\}}+\operatorname{sign}(x) \mathbb{1}_{\{|x| \geq 1\}}$. That is, for all $\theta \in \mathbb{R}$

$$
\mathbb{E}\left[e^{i \theta \tilde{L}_{1}}\right]=\exp \left(i \theta \eta+\int_{\mathbb{R}}\left(e^{i \theta x}-1-i \theta \tau(x)\right) \widetilde{v}(d x)\right) .
$$

In this situation the modulars and norms defined above become much more involved and harder to control, which is the main reason why we consider only symmetric Lévy motions as driving processes. Moreover, assumptions (A), (B1) and (B2) are not sufficient to guarantee the existence of the integral (1.2) if we consider non-symmetric Lévy processes, e.g. if $L_{t}=\eta t$ with $\eta \neq 0$. For more details, we refer to [26], Chapter 9.1. For our purposes, the following integrability criterion with respect to non-symmetric Lévy processes will suffice. For a predictable process $\left(F_{t}\right)_{t \in \mathbb{R}}$ define

$$
\Psi_{0, \tilde{L}}(F)=\int_{\mathbb{R}}\left|\int_{\mathbb{R}} \tau\left(u F_{s}\right)-\tau(u) F_{s} \widetilde{v}(d u)+\eta F_{S}\right| d s .
$$

Then, the condition

$$
\Phi_{0, \tilde{L}}(F)+\Psi_{0, \widetilde{L}}(F)<\infty \quad \text { almost surely }
$$

is sufficient for the integral $\int_{\mathbb{R}} F_{S} d \widetilde{L}_{s}$ to exist, and we write $F \in \mathbf{L}^{0}(d \widetilde{L})$. Indeed, this is a consequence of [26], Theorem 9.1.1 and pp. 217-218, combined with the estimate [30], Lemma 2.8.

\section{Proofs}

In this section, we present the proofs of our main results. The proof of (i) is divided into two parts and is similar to the proof of the corresponding result in [13]. First, we show the theorem under the assumption that $L$ is a compound Poisson process with jumps bounded away from zero in absolute value by some $a>0$. Thereafter, we argue that the contribution of the jumps of $L$ with absolute value $\leq a$ to the power variation becomes negligible as $a \rightarrow 0$. The proof of Theorem 1.1(ii) relies on freezing the intermittency $\sigma$ over small blocks and then deducing the result from [13], Theorem 1.1. A key step in the proof of Theorem 1.1(iii) is an application of a suitable stochastic Fubini result that we introduce in Section 4.3. 
Throughout the proofs, we denote all positive constants that do not depend on $n$ or $\omega$ by $C$, even though they may change from line to line. Similarly, we will denote by $K$ any positive random variable that does not depend on $n$, but may change from line to line. For a random variable $Y$ and $q>0$, we denote $\|Y\|_{q}=\mathbb{E}\left[|Y|^{q}\right]^{1 / q}$. We frequently use the notation

$$
g_{i, n}(s)=\sum_{j=0}^{k}(-1)^{j}\left(\begin{array}{l}
k \\
j
\end{array}\right) g((i-j) / n-s),
$$

which allows us to express the $k$ th order increments of $X$ as

$$
\Delta_{i, k}^{n} X=\int_{-\infty}^{i / n} g_{i, n}(s) \sigma_{s-} d L_{s}
$$

Recalling that $\left|g^{(k)}(s)\right| \leq C t^{\alpha-k}$ for all $s \in(0, \delta)$ and $\left|g^{(k)}\right|$ is decreasing on $(\delta, \infty)$ by assumption (A), Taylor expansion leads to the following important estimates.

Lemma 4.1. Suppose that Assumption (A) is satisfied. It holds that

$$
\begin{aligned}
& \left|g_{i, n}(s)\right| \leq C(i / n-s)^{\alpha} \quad \text { for } s \in[(i-k) / n, i / n], \\
& \left|g_{i, n}(s)\right| \leq C n^{-k}((i-k) / n-s)^{\alpha-k} \quad \text { for } s \in(i / n-\delta,(i-k) / n),
\end{aligned}
$$

and

$$
\begin{aligned}
& \left|g_{i, n}(s)\right| \leq C n^{-k}\left(\mathbb{1}_{[(i-k) / n-\delta, i / n-\delta]}(s)+g^{(k)}((i-k) / n-s) \mathbb{1}_{(-\infty,(i-k) / n-\delta)}(s)\right), \\
& \quad \text { for } s \in(-\infty, i / n-\delta] .
\end{aligned}
$$

Applying a standard localisation argument (cf. [7], Section 3) we can and will assume throughout the proofs that the process $\sigma$ is uniformly bounded by a constant on $[-\delta, \infty)$.

We conclude this subsection with a definition and some brief remarks on the Skorokhod $M_{1}$-topology. It was originally introduced by Skorokhod [33] by defining a metric on the completed graphs of càdlàg functions, where the completed graph of $f$ is defined as

$$
\Gamma_{f}=\left\{(x, t) \in \mathbb{R} \times \mathbb{R}_{+}: x=\alpha f(t-)+(1-\alpha) f(t), \text { for some } \alpha \in[0,1]\right\} .
$$

The $M_{1}$-topology is weaker as the more commonly used $J_{1}$-topology but still strong enough to make many important functionals, such as sup and inf, continuous. It can be shown that the stable convergence in Theorem 1.1 does not hold with respect to the $J_{1}$-topology. As $M_{1}$ is metrisable, it is entirely defined by characterising convergence of sequences, as we do in the following. A sequence $f_{n}$ of functions in $\mathbb{D}\left(\mathbb{R}_{+}, \mathbb{R}\right)$ converges to $f \in \mathbb{D}\left(\mathbb{R}_{+}, \mathbb{R}\right)$ with respect to the Skorokhod $M_{1}$-topology if and only if $f_{n}(t) \rightarrow f(t)$ for all $t$ in a dense subset of $[0, \infty)$, and for all $t_{\infty} \in[0, \infty)$ it holds that

$$
\lim _{\delta \downarrow} \limsup _{n \rightarrow \infty} \sup _{0 \leq t \leq t_{\infty}} w\left(f_{n}, t, \delta\right)=0 .
$$


Here, the oscillation function $w$ is defined as

$$
w(f, t, \delta)=\sup _{0 \vee(t-\delta) \leq t_{1}<t_{2}<t_{3} \leq(t+\delta) \wedge t_{\infty}}\left\{\left|f\left(t_{2}\right)-\left[f\left(t_{1}\right), f\left(t_{3}\right)\right]\right|\right\},
$$

where for $b<a$ the interval $[a, b]$ is defined to be $[b, a]$, and $|a-[b, c]|:=\inf _{d \in[b, c]}|a-d|$.

\subsection{Proof of Theorem 1.1(i)}

For the proof of Theorem 1.1(i), we follow the strategy from [13], Theorem 1.1(i). We assume first that $L$ is a compound Poisson process with jumps bounded in absolute value away from zero by some $a>0$. Later on, we argue that the small jumps of $L$ are asymptotically negligible. In order to show functional $\mathcal{F}$-stable convergence on $\mathbb{D}\left(\mathbb{R}_{+} ; \mathbb{R}\right)$ it is sufficient to show $\mathcal{F}$-stable convergence on $\mathbb{D}\left(\left[0, t_{\infty}\right] ; \mathbb{R}\right)$, for arbitrary but fixed $t_{\infty}>0$ (cf. [35], Chapter 3.3). Throughout this subsection, we therefore fix a $t_{\infty}>0$, and denote by $\mathbb{D}$ the space $\mathbb{D}\left(\left[0, t_{\infty}\right] ; \mathbb{R}\right)$ equipped with the Skorokhod $M_{1}$-topology, and by $\stackrel{\mathcal{L}_{M_{1}}-s}{\longrightarrow}$ the $\mathcal{F}$-stable convergence of $\mathbb{D}$-valued processes.

\subsubsection{Compound Poisson case}

Suppose that $\left(L_{t}\right)_{t \in \mathbb{R}}$ is a symmetric compound Poisson process with Lévy measure $v$, satisfying $v([-a, a])=0$ for some $a>0$. Let $0 \leq T_{1}<T_{2}<\cdots$ denote the jump times of $\left(L_{t}\right)_{t \geq 0}$ in increasing order. For $\varepsilon>0$, we define

$$
\begin{aligned}
\Omega_{\varepsilon}= & \left\{\omega \in \Omega: \text { for all } m \text { with } T_{m}(\omega) \in\left[0, t_{\infty}\right] \text { we have }\left|T_{m}(\omega)-T_{m-1}(\omega)\right|>\varepsilon\right. \\
& \text { and } \left.\Delta L_{s}(\omega)=0 \text { for all } s \in[-\varepsilon, 0]\right\} .
\end{aligned}
$$

We note that $\Omega_{\varepsilon} \uparrow \Omega$, as $\varepsilon \downarrow 0$. Letting

$$
M_{i, n, \varepsilon}:=\int_{i / n-\varepsilon}^{i / n} g_{i, n}(s) \sigma_{s-} d L_{s}, \quad \text { and } \quad R_{i, n, \varepsilon}:=\int_{-\infty}^{i / n-\varepsilon} g_{i, n}(s) \sigma_{s-} d L_{s},
$$

we have the decomposition $\Delta_{i, k}^{n} X=M_{i, n, \varepsilon}+R_{i, n, \varepsilon}$. It turns out that $M_{i, n, \varepsilon}$ is the asymptotically dominating term, whereas $R_{i, n, \varepsilon}$ is negligible as $n \rightarrow \infty$. We show that, on $\Omega_{\varepsilon}$,

$$
n^{\alpha p} \sum_{i=k}^{[n t]}\left|M_{i, n, \varepsilon}\right|^{p} \stackrel{\mathcal{L}_{M_{1}-s}}{\longrightarrow} Z_{t}, \quad \text { where } Z_{t}=\left|c_{0}\right|^{p} \sum_{m: T_{m} \in(0, t]}\left|\Delta L_{T_{m}} \sigma_{T_{m}-}\right|^{p} V_{m},
$$

where $\left(V_{m}\right)_{m \geq 1}$ are defined in Theorem 1.1(i). Denote by $i_{m}$ the random index such that $T_{m} \in$ $\left(\left(i_{m}-1\right) / n, i_{m} / n\right]$. Then, we have on $\Omega_{\varepsilon}$

$$
n^{\alpha p} \sum_{i=k}^{[n t]}\left|M_{i, n, \varepsilon}\right|^{p}=n^{\alpha p} \sum_{m: T_{m} \in(0,[n t] / n]}\left|\Delta L_{T_{m}} \sigma_{T_{m}-}\right|^{p}\left(\sum_{l=0}^{v_{t}^{m}}\left|g_{i_{m}+l, n}\left(T_{m}\right)\right|^{p}\right):=V_{t}^{n, \varepsilon},
$$


where the random index $v_{t}^{m}$ is defined as

$$
v_{t}^{m}=v_{t}^{m}(\varepsilon, n)= \begin{cases}{[\varepsilon n] \wedge\left([n t]-i_{m}\right)} & \text { if } T_{m}-\left([\varepsilon n]+i_{m}\right) / n>-\varepsilon, \\ ([\varepsilon n]-1) \wedge\left([n t]-i_{m}\right) & \text { if } T_{m}-\left([\varepsilon n]+i_{m}\right) / n \leq-\varepsilon .\end{cases}
$$

For the proof of (4.2) we first show stable convergence of the finite dimensional distributions of $V^{n, \varepsilon}$. Thereafter, we show that the sequence $\left(V^{n, \varepsilon}\right)_{n \geq 1}$ is tight and deduce the functional convergence $V^{n, \varepsilon} \stackrel{\mathcal{L}_{M_{1}}-s}{\longrightarrow} Z$.

Lemma 4.2. For $r \geq 1$ and $0 \leq t_{1}<\cdots<t_{r} \leq t_{\infty}$ we obtain on $\Omega_{\varepsilon}$ the $\mathcal{F}$-stable convergence

$$
\left(V_{t_{1}}^{n, \varepsilon}, \ldots, V_{t_{r}}^{n, \varepsilon}\right) \stackrel{\mathcal{L}-s}{\longrightarrow}\left(Z_{t_{1}}, \ldots, Z_{t_{r}}\right), \quad \text { as } n \rightarrow \infty .
$$

Proof. Let $\left(U_{i}\right)_{i \geq 1}$ be i.i.d. $\mathcal{U}([0,1])$-distributed random variables, defined on an extension $\left(\Omega^{\prime}, \mathcal{F}^{\prime}, \mathbb{P}^{\prime}\right)$ of the original probability space, independent of $\mathcal{F}$. By arguing as in [13], Section 5.1, we deduce for any $d \geq 1$ the $\mathcal{F}$-stable convergence

$$
\left\{n^{\alpha} g_{i_{m}+l, n}\left(T_{m}\right)\right\}_{l, m \leq d} \stackrel{\mathcal{L}-s}{\longrightarrow}\left\{c_{0} h_{k}\left(l+U_{m}\right)\right\}_{l, m \leq d}
$$

as $n \rightarrow \infty$, where $h_{k}$ is defined in (1.5). Defining

$$
\begin{aligned}
V_{t}^{n, \varepsilon, d} & :=n^{\alpha p} \sum_{m \leq d: T_{m} \in(0,[n t] / n]}\left|\Delta L_{T_{m}} \sigma_{T_{m}-}\right|^{p}\left(\sum_{l=0}^{d}\left|g_{i_{m}+l, n}\left(T_{m}\right)\right|^{p}\right), \\
Z_{t}^{d} & :=\left|c_{0}\right|^{p} \sum_{m \leq d: T_{m} \in(0, t]}\left|\Delta L_{T_{m}} \sigma_{T_{m}-}\right|^{p}\left(\sum_{l=0}^{d}\left|h_{k}\left(l+U_{m}\right)\right|^{p}\right),
\end{aligned}
$$

the continuous mapping theorem for stable convergence yields

$$
\left(V_{t_{1}}^{n, \varepsilon, d}, \ldots, V_{t_{r}}^{n, \varepsilon, d}\right) \stackrel{\mathcal{L}-s}{\longrightarrow}\left(Z_{t_{1}}^{d}, \ldots, Z_{t_{r}}^{d}\right), \quad \text { for } n \rightarrow \infty,
$$

for all $d \geq 1$. It follows by Lemma 4.1 for all $l$ with $k \leq l<[n \delta]$ that

$$
n^{\alpha p}\left|g_{i_{m}+l, n}\left(T_{m}\right)\right|^{p} \leq C|l-k|^{(\alpha-k) p},
$$

where we recall that $(\alpha-k) p<-1$. Consequently, we find a random variable $K>0$ such that for all $t \in\left[0, t_{\infty}\right]$

$$
\left|V_{t}^{n, \varepsilon, d}-V_{t}^{n, \varepsilon}\right| \leq K\left(\sum_{m>d: T_{m} \in\left[0, t_{\infty}\right]}\left|\Delta L_{T_{m}} \sigma_{T_{m}-}\right|^{p}+\sum_{m: T_{m} \in\left[0, t_{\infty}\right]} \sum_{l=v_{t}^{m} \wedge d}^{\infty}|l-k|^{(\alpha-k) p}\right) .
$$

By definition, the random index $v_{t}^{m}=v_{t}^{m}(n, \omega)$ satisfies $\liminf _{n \rightarrow \infty} v_{t}^{m}(n, \omega)=\infty$ for all $\omega$ with $T_{m}(\omega) \neq t$. Consequently, we obtain that $\limsup _{n \rightarrow \infty}\left|V_{t}^{n, \varepsilon, d}-V_{t}^{n, \varepsilon}\right| \rightarrow 0$ almost surely as 
$d \rightarrow \infty$. It follows that on $\Omega_{\varepsilon}$

$$
\limsup _{n \rightarrow \infty}\left\{\max _{t \in\left\{t_{1}, \ldots, t_{r}\right\}}\left|V_{t}^{n, \varepsilon}-V_{t}^{n, \varepsilon, d}\right|\right\} \rightarrow 0, \quad \text { almost surely, as } d \rightarrow \infty .
$$

By monotone convergence theorem we obtain that $\sup _{t \in\left[0, t_{\infty}\right]}\left|Z_{t}^{d}-Z_{t}\right| \rightarrow 0$ as $d \rightarrow \infty$. Together with (4.3) and (4.4), this implies the statement of the lemma by a standard approximation argument, see, for example, [16], Theorem 3.2.

Recall that the stable convergence $V^{n, \varepsilon} \stackrel{\mathcal{L}_{M_{1}}-s}{\longrightarrow} Z$ is equivalent to the joint convergence in law $\left(V^{n, \varepsilon}, Y\right) \stackrel{\mathcal{L}}{\longrightarrow}(Z, Y)$ for all $\mathcal{F}$-measurable random variables $Y$, cf. [25], Proposition 5.33. Consequently, Lemma 4.2 and the following result together with Prokhorov's theorem imply (4.2), where we recall that $\left(\mathbb{D}\left(\left[0, t_{\infty}\right]\right), M_{1}\right)$ is a Polish space.

Lemma 4.3. The sequence $\left(V^{n, \varepsilon}\right)_{n \geq 1}$ of $\left(\mathbb{D}\left(\left[0, t_{\infty}\right]\right), M_{1}\right)$-valued random variables is tight.

Proof. The claim follows from [35], Theorem 12.12.3, if we verify that $\left(V^{n, \varepsilon}\right)_{n \geq 1}$ satisfies the conditions of the theorem. Condition (i) follows since the processes $V^{n, \varepsilon}$ are increasing in $t$ and from tightness of $\left\{V_{t_{\infty}}^{n, \varepsilon}\right\}_{n \in \mathbb{N}}$, which follows from Lemma 4.2. For condition (ii) we need to verify that for all $\zeta, \xi>0$ there is an $\eta>0$ such that

$$
\mathbb{P}\left(\sup _{t \in\left[0, t_{\infty}\right]} w\left(V^{n, \varepsilon}, t, \eta\right)>\xi\right) \leq \zeta, \quad \text { for all } n,
$$

where the oscillation function $w$ was defined in (4.1). This follows since the processes $V^{n, \varepsilon}$ are increasing, and consequently $w\left(V^{n, \varepsilon}, t, \eta\right)=0$ for all $n$, all $t$ and all $\eta$.

This concludes the proof of (4.2). Next, we show that

$$
n^{\alpha p} \sum_{i=k}^{\left[n t_{\infty}\right]}\left|R_{i, n, \varepsilon}\right|^{p} \stackrel{\mathbb{P}}{\longrightarrow} 0 .
$$

Recalling that $\alpha<k-1 / p$, it is sufficient to show that

$$
\sup _{n \in \mathbb{N}} \sup _{i \in\left\{k, \ldots,\left[n t_{\infty}\right]\right\}} n^{k}\left|R_{i, n, \varepsilon}\right|<\infty, \quad \text { almost surely. }
$$

It follows from Lemma 4.1 that

$$
n^{k}\left|g_{i, n}(s) \sigma_{s-}\right| \leq C\left(\mathbb{1}_{\left[-\delta, t_{\infty}\right]}(s)+\left|g^{(k)}(-s) \sigma_{s-}\right| \mathbb{1}_{(-\infty,-\delta)}(s)\right):=\psi_{s} .
$$

Let $\widetilde{L}=\left(\widetilde{L}_{t}\right)_{t \in \mathbb{R}}$ denote the process defined by $\widetilde{L}_{0}=0$ and $\widetilde{L}_{t}-\widetilde{L}_{u}$ is the total variation of $v \mapsto$ $L_{v}$ on $(u, t]$ for all $u<t$. Since $L$ is a compound Poisson process, the process $\widetilde{L}$ is well-defined, finite and it follows from [32], Theorem 21.9 , that $\widetilde{L}$ is a Lévy process with Lévy measure 
$\widetilde{v}=2 v_{\mid \mathbb{R}_{+}}$and shift parameter $\eta$ with respect to the truncation function $\tau: x \mapsto x \mathbb{1}_{\{|x|<1\}}+$ $\operatorname{sign}(x) \mathbb{1}_{\{|x| \geq 1\}}$ given by $\eta=\int_{\mathbb{R}} \tau(x) \widetilde{v}(d x)$. Next, we use the following estimate:

$$
n^{k}\left|R_{i, n, \varepsilon}\right| \leq \int_{\left(-\infty, \frac{i}{n}-\varepsilon\right]} n^{k}\left|g_{i, n}(s) \sigma_{s-}\right| d \widetilde{L}_{s} \leq \int_{\mathbb{R}} \psi_{s} d \widetilde{L}_{s} .
$$

The right-hand side is finite almost surely due to the following Lemma 4.4, and the proof of (4.5) is complete.

Lemma 4.4. Let $L$ be a symmetric compound Poisson process with Lévy measure $v$ satisfying $v([-a, a])=0$ for some $a \in(0,1]$ and let $\widetilde{L}$ and $\psi$ be given as above. Suppose, in addition, that (B1) is satisfied. Then the stochastic integral $\int_{\mathbb{R}} \psi_{s} d \widetilde{L}_{s}$ exists and is finite almost surely.

Proof. To show that the stochastic integral $\int_{\mathbb{R}} \psi_{s} d \widetilde{L}_{s}$ is well-defined it is enough to prove that $\Phi_{0, \widetilde{L}}(\psi)+\Psi_{0, \widetilde{L}}(\psi)<\infty$ almost surely (see (3.7) of Section 3). For some $\beta^{\prime}>\beta$, we have from (B1) that

$$
\int_{\mathbb{R}}\left|\psi_{s}\right|^{\theta} \mathbb{1}_{\left\{\left|\psi_{s}\right| \leq 1\right\}}+\left|\psi_{s}\right|^{\beta^{\prime}} \mathbb{1}_{\left\{\left|\psi_{s}\right|>1\right\}} d s<\infty, \quad \text { a.s. }
$$

This implies that $\Phi_{0, \widetilde{L}}(\psi)<\infty$ almost surely (cf. Remark 3.3). Next, we note that

$$
\begin{aligned}
\Psi_{0, L}(\psi) & =\int_{\mathbb{R}}\left|\int_{\mathbb{R}} \tau\left(x \psi_{s}\right)-\tau(x) \psi_{s} \widetilde{v}(d x)+\eta \psi_{s}\right| d s \\
& =\int_{\mathbb{R}}\left|\int_{\mathbb{R}} \tau\left(x \psi_{s}\right) \widetilde{v}(d x)\right| d s,
\end{aligned}
$$

where the second equality follows by definition of $\eta$ above. Hence, to show that $\Psi_{0, L}(\psi)<$ $\infty$ almost surely, it suffices according to (B1) to derive the following estimate. There exists a constant $C>0$ such that for all $u \in \mathbb{R}$

$$
\int_{\mathbb{R}}|\tau(u x)| \widetilde{v}(d x) \leq C\left(|u|^{\rho} \mathbb{1}_{\{|u| \leq 1\}}+\mathbb{1}_{\{|u|>1\}}\right),
$$

where $\rho$ is as in assumption (B1). By the definitions of $\tau$ and $\widetilde{v}$ we have that

$$
\int_{\mathbb{R}}|\tau(u x)| \widetilde{v}(d x)=|u| \int_{\left\{|x| \leq|u|^{-1}\right\}}|x| v(d x)+v(x \in \mathbb{R}:|x u|>1) .
$$

We recall that $\limsup _{t \rightarrow \infty} v([t, \infty)) t^{\theta}<\infty$. Since $v$ is finite, there exists $C_{0}>0$ such that $v([t, \infty)) \leq C_{0} / t^{\theta}$ for all $t \geq a$. Consequently, we obtain for all $t \geq a$ and $f(u)=\mathbb{1}_{[t, \infty)}(u)$

$$
\int_{a}^{\infty} f(x) v(d x) \leq \frac{C_{0}}{\theta} \int_{a}^{\infty} f(x) x^{-\theta-1} d x .
$$


By monotone approximation, the inequality remains valid for all nondecreasing $f:[a, \infty) \rightarrow$ $\mathbb{R}_{+}$. Therefore, the first term on the right-hand side of (4.7) is bounded by

$$
\begin{aligned}
|u| \int_{\left\{|x| \leq|u|^{-1}\right\}}|x| v(d x) & \leq\left(C_{0} / \theta\right) \mathbb{1}_{\left\{|u| \leq a^{-1}\right\}}|u| \int_{a}^{|u|^{-1}}|x|^{-\theta} d x \\
& \leq C \mathbb{1}_{\left\{|u| \leq a^{-1}\right\}} \begin{cases}|u|^{\theta}, & \theta<1 \\
|u|(\log (1 /|u|)+\log (1 / a)), & \theta=1 \\
|u|, & \theta>1 .\end{cases}
\end{aligned}
$$

For the second term on the right-hand side of (4.7), we use the following estimate

$$
v(x \in \mathbb{R}:|x u|>1) \leq C\left(\mathbb{1}_{\{|u|>1\}}+\left(|u|^{-1}\right)^{-\theta} \mathbb{1}_{\{|u| \leq 1\}}\right)=C\left(\mathbb{1}_{\{|u|>1\}}+|u|^{\theta} \mathbb{1}_{\{|u| \leq 1\}}\right)
$$

for all $u \in \mathbb{R}$, which completes the proof of (4.6) and hence of the lemma.

Recalling the decomposition $\Delta_{i, k}^{n} X=M_{i, n, \varepsilon}+R_{i, n, \varepsilon}$ we obtain by Minkowski's inequality

$$
\sup _{t \in\left[0, t_{\infty}\right]}\left|\left(n^{\alpha p} V(p ; k)_{t}^{n}\right)^{\frac{1}{p}}-\left(n^{\alpha p} \sum_{i=k}^{[n t]}\left|M_{i, n, \varepsilon}\right|^{p}\right)^{\frac{1}{p}}\right| \leq\left(n^{\alpha p} \sum_{i=k}^{\left[n t_{\infty}\right]}\left|R_{i, n, \varepsilon}\right|^{p}\right)^{\frac{1}{p}} .
$$

Therefore, by virtue of (4.2) and (4.5), we conclude that

$$
n^{\alpha p} V(p ; k)_{t}^{n} \stackrel{\mathcal{L}_{M_{1}}-s}{\longrightarrow} Z_{t} \quad \text { on } \Omega_{\varepsilon} .
$$

By letting $\varepsilon \rightarrow 0$, we conclude that Theorem 1.1(i) holds, when $L$ is a compound Poisson process with jumps bounded away from 0 .

\subsubsection{Decomposition into big and small jumps}

In this section, we extend the proof of Theorem 1.1(i) to general symmetric Lévy processes $\left(L_{t}\right)_{t \in \mathbb{R}}$. We need the following preliminary result.

Lemma 4.5. Let $q \geq 1$ and $a \in(0,1]$. The function

$$
\xi(y)=\int_{-a}^{a}|y x|^{2} \mathbb{1}_{\{|y x| \leq 1\}}+|y x|^{q} \mathbb{1}_{\{|y x|>1\}} v(d x)
$$

satisfies $|\xi(y)| \leq C\left(|y|^{2} \mathbb{1}_{\{|y \leq 1|\}}+|y|^{\beta^{\prime} \vee q_{1}} \mathbb{1}_{\{|y>1|\}}\right)$ for any $\beta^{\prime}>\beta$, where $C$ does not depend on a.

Proof. Use the decomposition $\xi=\xi_{1}+\xi_{2}$ with

$$
\xi_{1}(y)=\int_{-a}^{a}|y x|^{2} \mathbb{1}_{\{|y x| \leq 1\}} \nu(d x), \quad \text { and } \quad \xi_{2}(y)=\int_{-a}^{a}|y x|^{q} \mathbb{1}_{\{|y x|>1\}} v(d x) .
$$


We obtain

$$
\xi_{1}(y) \mathbb{1}_{\{|y| \leq 1\}} \leq|y|^{2} \int_{-1}^{1} x^{2} v(d x) \mathbb{1}_{\{|y| \leq 1\}},
$$

and $\xi_{1}(y) \mathbb{1}_{\{|y|>1\}} \leq C|y|^{\beta^{\prime} \vee q} \mathbb{1}_{\{|y|>1\}}$ follows from (3.4), showing that $\xi_{1}$ satisfies the estimate given in the lemma. For $q>\beta$, we obtain

$$
\xi_{2}(y)=2|y|^{q} \mathbb{1}_{\{|y|>1 / a\}} \int_{1 /|y|}^{a}|x|^{q} v(d x) \leq C|y|^{q} \mathbb{1}_{\{|y| \geq 1\}} .
$$

If $q \leq \beta$, we have similarly for any $\beta^{\prime}>\beta$

$$
\xi_{2}(y) \leq 2|y|^{\beta^{\prime}} \mathbb{1}_{\{|y|>1 / a\}} \int_{1 /|y|}^{a}|x|^{\beta^{\prime}} v(d x) \leq C|y|^{\beta^{\prime}} \mathbb{1}_{\{|y| \geq 1\}},
$$

which completes the proof.

Now, given a general symmetric Lévy process $\left(L_{t}\right)_{t \in \mathbb{R}}$, consider for $a>0$ the compound Poisson process $\left(L_{t}^{>a}\right)_{t \in \mathbb{R}}$ defined by

$$
L_{0}^{>a}=0, \quad L_{t}^{>a}-L_{s}^{>a}=\sum_{s<u \leq t} \Delta L_{u} \mathbb{1}_{\left\{\left|\Delta L_{u}\right|>a\right\}} .
$$

Moreover, let $\left(L_{t}^{\leq a}\right)_{t \in \mathbb{R}}$ denote the Lévy process $\left(L_{t}-L_{t}^{>a}\right)_{t \in \mathbb{R}}$. The key result of this section is showing that

$$
\limsup _{n \rightarrow \infty}\left\|n^{\alpha p} \sum_{i=k}^{\left[n t_{\infty}\right]}\left|\int_{-\infty}^{i / n} g_{i, n}(s) \sigma_{s-} d L_{s}^{\leq a}\right|^{p}\right\|_{1} \rightarrow 0, \quad \text { as } a \rightarrow 0 .
$$

We make the decomposition

$$
\int_{-\infty}^{i / n} g_{i, n}(s) \sigma_{s-} d L_{s}^{\leq a}=A_{i, n}+B_{i, n},
$$

where

$$
A_{i, n}=\int_{-\delta}^{i / n} g_{i, n}(s) \sigma_{s-} d L_{s}^{\leq a} \quad \text { and } \quad B_{i, n}=\int_{-\infty}^{-\delta} g_{i, n}(s) \sigma_{s-} d L_{s}^{\leq a} .
$$

Lemma 3.1 shows that

$$
\begin{aligned}
\left\|n^{\alpha p} \sum_{i=k}^{\left[n t_{\infty}\right]}\left|A_{i, n}\right|^{p}\right\|_{1} & =n^{-1} \sum_{i=k}^{\left[n t_{\infty}\right]}\left\|\int_{-\delta}^{i / n} n^{\alpha+1 / p} g_{i, n}(s) \sigma_{s-} d L_{s}^{\leq a}\right\|_{p}^{p} \\
& \leq C n^{-1} \sum_{i=k}^{\left[n t_{\infty}\right]} \mathbb{E}\left[\left\|F^{i, n}\right\|_{p, L \leq a}^{p}\right]
\end{aligned}
$$


where the process $\left(F_{t}^{i, n}\right)_{t \in \mathbb{R}}$ is defined as $F_{t}^{i, n}=n^{\alpha+1 / p} g_{i, n}(t) \mathbb{1}_{(-\delta, i / n]}(t) \sigma_{t-}$. Since the random variable $\sup _{t \in[-\delta, \infty)}\left|\sigma_{t}\right|$ is uniformly bounded, we obtain by (3.6) and [13], equation (4.23),

$$
\begin{aligned}
\mathbb{E}\left[\left\|F^{i, n}\right\|_{p, L \leq a}^{p}\right] & \leq C\left\|n^{\alpha+1 / p} g_{i, n} \mathbb{1}_{[-\delta, i / n]}\right\|_{p, L \leq a}^{p} \\
& \leq C\left|\Phi_{p, L \leq a}\left(n^{\alpha+1 / p} g_{k, n}\right)\right|^{p / 2} \vee\left|\Phi_{p, L \leq a}\left(n^{\alpha+1 / p} g_{k, n}\right)\right| \\
& \leq C\left(\int_{|x| \leq a}|x|^{p}+x^{2} v(d x)\right)^{p / 2} \vee\left(\int_{|x| \leq a}|x|^{p}+x^{2} v(d x)\right)
\end{aligned}
$$

for all $n \in \mathbb{N}$ and $i \in\left\{k, \ldots,\left[n t_{\infty}\right]\right\}$. Since $p>\beta$ by assumption, we conclude that

$$
\limsup _{n \rightarrow \infty}\left\|n^{\alpha p} \sum_{i=k}^{\left[n t_{\infty}\right]}\left|A_{i, n}\right|^{p}\right\|_{1} \rightarrow 0, \quad \text { as } a \rightarrow 0
$$

Next, we show that for all $a>0$

$$
\limsup _{n \rightarrow \infty}\left\|n^{\alpha p} \sum_{i=k}^{\left[n t_{\infty}\right]}\left|B_{i, n}\right|^{p}\right\|_{1}=0
$$

Introducing the processes $\left(Y_{t}^{i, n}\right)_{t \in \mathbb{R}}$ and $\left(Y_{t}\right)_{t \in \mathbb{R}}$ defined as

$$
Y_{t}^{i, n}=n^{\alpha+1 / p} g_{i, n}(t) \sigma_{t-\mathbb{1}_{(-\infty,-\delta]}}(t), \quad \text { and } \quad Y_{t}=\mid g^{(k)}(-t) \sigma_{t-\mathbb{1}_{(-\infty,-\delta]}(t) \mid,}
$$

we obtain by Lemma 3.1 that

$$
\left\|n^{\alpha p} \sum_{i=k}^{\left[n t_{\infty}\right]}\left|B_{i, n}\right|^{p}\right\|_{1} \leq C n^{-1} \sum_{i=k}^{\left[n t_{\infty}\right]} \mathbb{E}\left[\left\|Y^{i, n}\right\|_{p, L \leq a}^{p}\right]
$$

Moreover, recalling that $\left|g^{(k)}\right|$ is decreasing on $(\delta, \infty)$, an application of Lemma 4.1 shows that

$$
\mathbb{E}\left[\left\|Y^{i, n}\right\|_{p, L \leq a}^{p}\right] \leq n^{p(\alpha+1 / p-k)} \mathbb{E}\left[\|Y\|_{p, L \leq a}^{p}\right],
$$

for all $i \in\{k, \ldots, n\}$. Since $\alpha+1 / p-k<0$, equation (4.10) follows if $\mathbb{E}\left[\|Y\|_{p, L \leq a}^{p}\right]<\infty$. Applying the estimate (3.6) shows that this is satisfied if $\mathbb{E}\left[\Phi_{p, L \leq a}^{1 \vee \frac{p}{2}}(Y)\right]<\infty$, which is a consequence of (B1) and Lemma 4.5, where we used that $p>\beta$. Now, (4.8) follows from (4.9) and (4.10).

We can complete the proof of Theorem 1.1(i) by combining (4.8) with the results of Section 4.1.1. To this end, let

$$
X_{t}^{>a}:=\int_{-\infty}^{t}\left(g(t-s)-g_{0}(-s)\right) \sigma_{s-} d L_{s}^{>a}, \quad X_{t}^{\leq a}:=\int_{-\infty}^{t}\left(g(t-s)-g_{0}(-s)\right) \sigma_{s-} d L_{s}^{\leq a},
$$


and let $T_{m}^{>a}=T_{m}$ if $\left|\Delta L_{T_{m}}\right|>a$, and $T_{m}^{>a}=\infty$ else. The results of Section 4.1.1 show that

$$
n^{\alpha p} V\left(X^{>a}, p ; k\right)_{t}^{n} \stackrel{\mathcal{L}_{M_{1}}-s}{\longrightarrow} Z_{t}^{>a}:=\sum_{m: T_{m}^{>a} \in(0, t]}\left|\Delta L_{T_{m}^{>a}} \sigma_{T_{m}^{>a}-\mid}\right|^{p} V_{m}
$$

for all $a>0$, where $V\left(X^{>a}, p ; k\right)_{t}^{n}$ denotes the power variation of the process $X^{>a}$. Making the decomposition

$$
\begin{aligned}
& \left(n^{\alpha p} V(p ; k)_{t}^{n}\right)^{1 / p} \\
& \quad=\left(n^{\alpha p} V\left(X^{>a}, p ; k\right)_{t}^{n}\right)^{1 / p}+\left(\left(n^{\alpha p} V(p ; k)_{t}^{n}\right)^{1 / p}-\left(n^{\alpha p} V\left(X^{>a}, p ; k\right)_{t}^{n}\right)^{1 / p}\right) \\
& \quad:=U_{t}^{n,>a}+U_{t}^{n, \leq a},
\end{aligned}
$$

we have by Minkowski's inequality

$$
\lim _{a \rightarrow 0} \limsup _{n \rightarrow \infty} \mathbb{P}\left(\sup _{t \in\left[0, t_{\infty}\right]}\left|U_{t}^{n, \leq a}\right|>\varepsilon\right) \leq \lim _{a \rightarrow 0} \limsup _{n \rightarrow \infty} \mathbb{P}\left(n^{\alpha p} V\left(X^{\leq a}, p ; k\right)_{t_{\infty}}^{n}>\varepsilon^{p}\right)=0,
$$

for all $\varepsilon>0$, which follows easily from (4.8). Since $U_{t}^{n,>a} \stackrel{\mathcal{L}_{M_{1}}-s}{\longrightarrow} Z_{t}^{>a}$ as $n \rightarrow \infty$, and $\sup _{t \in\left[0, t_{\infty}\right]}\left|Z_{t}^{>a}-Z_{t}\right| \rightarrow 0$ almost surely, as $a \rightarrow 0$, Theorem 1.1(i) follows from [16], Theorem 3.2.

Remark 4.6. A popular technique for proving limit theorems for volatility modulated processes is to freeze the volatility over blocks of length $1 / n$ and derive a limit theorem for the resulting simpler process. However, in the framework of Theorem 1.1(i) this approach is not applicable, since the power variations of the two processes are not asymptotically equivalent if $\sigma$ and $L$ jump at the same times.

\subsection{Proof of Theorem 1.1(ii)}

Since $t \mapsto V(p ; k)_{t}^{n}$ is increasing and the limiting function is continuous, uniform convergence on compact sets in probability follows if we show

$$
n^{-1+p(\alpha+1 / \beta)} V(p ; k)_{t}^{n} \stackrel{\mathbb{P}}{\longrightarrow} m_{p} \int_{0}^{t}\left|\sigma_{s}\right|^{p} d s
$$

for a fixed $t>0$, which we will do in the following. A crucial step in the proof is to show that the asymptotic behavior of the power variation does not change if we replace $\Delta_{i, k}^{n} X$ in (1.3) by $\sigma_{(i-k) / n} \Delta_{i, k}^{n} G$, where the process $\left(G_{t}\right)_{t \geq 0}$ is defined as the integral in (1.2) with $\sigma \equiv 1$. Note that Assumption (A) ensures that $G$ is well-defined. Thereafter, we divide the interval $[0, t]$ into subblocks of size $1 / l$ and freeze $\sigma$ at the beginning of each block. The limiting power variation for the resulting process can then be derived by applying part (ii) of [13], Theorem 1.1, on every block. The proof of Theorem 1.1(ii) is then completed by letting $l \rightarrow \infty$. The following lemma 
plays an important role for replacing $\Delta_{i, k}^{n} X$ in (1.3) by $\sigma_{(i-k) / n} \Delta_{i, k}^{n} G$. Here and in the following we denote by $v_{\sigma}$ the modulus of continuity of $\sigma$ defined as

$$
v_{\sigma}(s, \eta)=\sup \left\{\left|\sigma_{s}-\sigma_{r}\right|: r \in[s-\eta, s+\eta]\right\}
$$

Lemma 4.7. Let $\left(\sigma_{t}\right)_{t \in \mathbb{R}}$ be a process with càdlàg or càglàd sample paths that is uniformly bounded on $[-\delta, \infty)$. For any $\alpha, q \in(0, \infty)$ we have

$$
\lim _{\varepsilon \rightarrow 0}\left[\limsup _{n \rightarrow \infty}\left(\frac{1}{n} \sum_{i=k}^{[n t]}\left\|v_{\sigma}(i / n, \varepsilon)\right\|_{q}^{\alpha}\right)\right]=0
$$

Proof. Since $v_{\sigma}$ is bounded and $x \mapsto x^{\alpha}$ is locally Lipschitz for $\alpha>1$, we may assume w.l.o.g. that $\alpha \leq 1$ and $q \geq 1$. For $\kappa>0$ we use the decomposition $\sigma=\sigma^{<\kappa}+\sigma^{\geq \kappa}$, where

$$
\sigma_{s}^{\geq \kappa}=\sum_{-\delta<u \leq s} \Delta \sigma_{u} \mathbb{1}_{\left\{\left|\Delta \sigma_{u}\right| \geq \kappa\right\}}
$$

and $\sigma_{s}^{<\kappa}=\sigma_{s}-\sigma_{s}^{\geq \kappa}$. Even though $\sigma$ is uniformly bounded on $[-\delta, \infty), \sigma^{\geq \kappa}$ and $\sigma^{<\kappa}$ might not be. For this reason, we introduce the sets

$$
\begin{aligned}
\Omega_{m}:= & \left\{\omega:\left|\sigma_{s}^{<\kappa}(\omega)\right|+\left|\sigma_{s}^{\geq \kappa}(\omega)\right| \leq m \text { for all } s \in[-\delta, t+\delta],\right. \\
& \text { and } \left.\sigma^{\geq \kappa}(\omega) \text { has less than } m \text { jumps in }[-\delta, t+\delta]\right\} .
\end{aligned}
$$

Note that $\Omega_{m} \uparrow \Omega$, as $m \rightarrow \infty$. By triangular inequality, we have $v_{\sigma}(s, \eta) \leq v_{\sigma<\kappa}(s, \eta) \mathbb{1}_{\Omega_{m}}+$ $v_{\sigma \geq k}(s, \eta) \mathbb{1}_{\Omega_{m}}+C \mathbb{1}_{\Omega_{m}^{\mathrm{c}}}$ for all $s \in[0, t], \eta<\delta$ and $m \geq 1$. Since $\mathbb{P}\left(\Omega_{m}^{\mathrm{c}}\right) \rightarrow 0$ as $m \rightarrow \infty$, we can choose $m$ sufficiently large such that

$$
\frac{1}{n} \sum_{i=k}^{[n t]}\left\|v_{\sigma}(i / n, \varepsilon)\right\|_{q}^{\alpha} \leq \frac{1}{n} \sum_{i=k}^{[n t]}\left(\left\|v_{\sigma<\kappa}(i / n, \varepsilon) \mathbb{1}_{\Omega_{m}}\right\|_{q}^{\alpha}+\left\|v_{\sigma \geq \kappa}(i / n, \varepsilon) \mathbb{1}_{\Omega_{m}}\right\|_{q}^{\alpha}\right)+\kappa
$$

for all $n \in \mathbb{N}$ and $\varepsilon>0$. We show that

$$
\limsup _{\varepsilon \rightarrow 0} \limsup _{n \rightarrow \infty}\left(\frac{1}{n} \sum_{i=k}^{[n t]}\left\|v_{\sigma}<\kappa(i / n, \varepsilon) \mathbb{1}_{\Omega_{m}}\right\|_{q}^{\alpha}\right) \leq 2 \kappa^{\alpha} .
$$

In order to do so, we assume the existence of sequences $\left(\varepsilon_{l}\right),\left(n_{l}\right),\left(i_{l}\right)$ with $\varepsilon_{l} \rightarrow 0, n_{l} \rightarrow \infty$ and $i_{l} \in\left\{1, \ldots,\left[t n_{l}\right]\right\}$ such that

$$
\left\|v_{\sigma<\kappa}\left(i_{l} / n_{l}, \varepsilon_{l}\right) \mathbb{1}_{\Omega_{m}}\right\|_{q}^{\alpha}>2 \kappa^{\alpha}
$$

for all $l$, and derive a contradiction. Since $\left(i_{l} / n_{l}\right)_{l \geq 1}$ is a bounded sequence we may assume that $i_{l} / n_{l}$ converges to some $s_{0} \in[0, t]$ by considering a suitable subsequence $\left(l_{k}\right)_{k \geq 1}$. For all $\omega \in$ $\Omega_{m}$ it holds that $\lim _{\gamma \rightarrow 0} v_{\sigma<\kappa}\left(s_{0}, \gamma\right)=\left|\Delta \sigma_{s_{0}}^{<\kappa}\right| \leq \kappa$. Therefore, by the dominated convergence theorem, we can find a $\gamma>0$ such that $\left\|v_{\sigma<\kappa}\left(s_{0}, \gamma\right) \mathbb{1}_{\Omega_{m}}\right\|_{q}^{\alpha} \leq 2 \kappa^{\alpha}$. This is a contradiction to 
(4.13), since for sufficiently large $l$ we have $\left[i_{l} / n_{l}-\varepsilon_{l}, i_{l} / n_{l}+\varepsilon_{l}\right] \subset\left[s_{0}-\gamma, s_{0}+\gamma\right]$. This completes the proof of (4.12). Next, we show that

$$
\lim _{\varepsilon \rightarrow 0} \limsup _{n \rightarrow \infty}\left(\frac{1}{n} \sum_{i=k}^{[n t]}\left\|v_{\sigma \geq \kappa}(i / n, \varepsilon) \mathbb{1}_{\Omega_{m}}\right\|_{q}^{\alpha}\right)=0 .
$$

Recalling that $q / \alpha \geq 1$, an application of Jensen's inequality yields

$$
\frac{1}{n} \sum_{i=k}^{[n t]}\left\|v_{\sigma \geq \kappa}(i / n, \varepsilon) \mathbb{1}_{\Omega_{m}}\right\|_{q}^{\alpha} \leq\left\|t^{q / \alpha-1} \frac{1}{n} \sum_{i=k}^{[n t]}\left(v_{\sigma \geq \kappa}(i / n, \varepsilon) \mathbb{1}_{\Omega_{m}}\right)^{q}\right\|_{1}^{\alpha / q},
$$

for all $n \in \mathbb{N}, \varepsilon>0$. Now, (4.14) follows from the estimate

$$
\frac{1}{n} \sum_{i=k}^{[n t]}\left(v_{\sigma \geq \kappa}(i / n, \varepsilon) \mathbb{1}_{\Omega_{m}}\right)^{q} \leq \sup _{s \in[-\delta, t+\delta]}\left|\Delta \sigma_{s}^{\geq \kappa}\right|^{q} N \mathbb{1}_{\Omega_{m}} 2(\varepsilon) \leq C m^{q+1}(\varepsilon),
$$

for all $n \in \mathbb{N}$. Here $N=N(\omega)$ denotes the number of jumps of $\sigma^{\geq \kappa}$ in $[-\delta, t+\delta]$. Using (4.12) and (4.14), the lemma now follows from (4.11) by letting $\kappa \rightarrow 0$.

The proof of Theorem 1.1(ii) heavily relies on the estimate given in Lemma 3.2. This lemma assumes the role that Itô's isometry typically plays in proofs of limit theorems for stochastic integral processes driven by a Brownian motion. In order to apply Lemma 3.2, the following estimates will be crucial.

Lemma 4.8. Suppose that Assumptions (A) and (B2) hold, and assume that $\alpha+1 / \beta<k$. For $\varepsilon>0$ with $\varepsilon \leq \delta$ there is a constant $C>0$ such that

$$
\begin{aligned}
& \mathbb{E}\left[\int_{\frac{i}{n}-\varepsilon}^{\frac{i}{n}}\left|g_{i, n}(s) \sigma_{s-}\right|^{\beta} d s\right]+\int_{\frac{i}{n}-\varepsilon}^{\frac{i}{n}}\left|g_{i, n}(s)\right|^{\beta} d s \leq C n^{-\alpha \beta-1}, \quad \text { and } \\
& \mathbb{E}\left[\int_{-\infty}^{\frac{i}{n}-\varepsilon}\left|g_{i, n}(s) \sigma_{s-}\right|^{\beta} d s\right]+\int_{-\infty}^{\frac{i}{n}-\varepsilon}\left|g_{i, n}(s)\right|^{\beta} d s \leq C n^{-k \beta},
\end{aligned}
$$

for all $i \in\{k, \ldots, n\}$.

Proof. By Lemma 4.1, we have that

$$
\begin{aligned}
& \left|g_{i, n}(s)\right|^{\beta} \mathbb{1}_{[i / n-\varepsilon, i / n]}(s) \\
& \quad \leq C\left((i / n-s)^{\alpha \beta} \mathbb{1}_{[(i-k) / n, i / n]}(s)+n^{-k \beta}((i-k) / n-s)^{(\alpha-k) \beta} \mathbb{1}_{[i / n-\varepsilon,(i-k) / n]}(s)\right) .
\end{aligned}
$$

Recalling that $\sigma$ is bounded on $[-\delta, \infty)$, the first inequality follows by calculating the integral of the right-hand side. The second inequality is a direct consequence of Lemma 4.1 and Assumptions (A) and (B2). 
A crucial step in the proof of Theorem 1.1(ii) is showing that

$$
n^{-1+p(\alpha+1 / \beta)} \sum_{i=k}^{[n t]}\left\|\Delta_{i, k}^{n} X-\sigma_{(i-k) / n} \Delta_{i, k}^{n} G\right\|_{p}^{p} \rightarrow 0
$$

as $n \rightarrow \infty$, where the process $\left(G_{t}\right)_{t \geq 0}$ is defined as the integral in (1.2) with $\sigma \equiv 1$. We fix some $\varepsilon>0$ and make the decomposition

$$
\Delta_{i, k}^{n} X-\sigma_{(i-k) / n} \Delta_{i, k}^{n} G=A_{i}^{n, \varepsilon}+B_{i}^{n, \varepsilon}+C_{i}^{n, \varepsilon},
$$

where

$$
\begin{aligned}
A_{i}^{n, \varepsilon} & =\int_{i / n-\varepsilon}^{i / n} g_{i, n}(s)\left(\sigma_{s-}-\sigma_{i / n-\varepsilon}\right) d L_{s}, \\
B_{i}^{n, \varepsilon} & =\left(\sigma_{i / n-\varepsilon}-\sigma_{(i-k) / n}\right) \int_{i / n-\varepsilon}^{i / n} g_{i, n}(s) d L_{s}, \\
C_{i}^{n, \varepsilon} & =\int_{-\infty}^{i / n-\varepsilon} g_{i, n}(s) \sigma_{s-} d L_{s}-\sigma_{(i-k) / n} \int_{-\infty}^{i / n-\varepsilon} g_{i, n}(s) d L_{s} .
\end{aligned}
$$

We deduce (4.15) by showing that

$$
\lim _{\varepsilon \rightarrow 0} \limsup _{n \rightarrow \infty}\left(n^{-1+p(\alpha+1 / \beta)} \sum_{i=k}^{[n t]}\left\|A_{i}^{n, \varepsilon}\right\|_{p}^{p}\right)=0,
$$

and the same for $B_{i}^{n, \varepsilon}$ and $C_{i}^{n, \varepsilon}$, respectively. For $A_{i}^{n, \varepsilon}$ we obtain by Lemma 3.2

$$
\begin{aligned}
& n^{-1+p(\alpha+1 / \beta)} \sum_{i=k}^{[n t]}\left\|A_{i}^{n, \varepsilon}\right\|_{p}^{p} \\
& \leq C n^{-1+p(\alpha+1 / \beta)} \sum_{i=k}^{[n t]}\left\{\mathbb{E}\left[\int_{i / n-\varepsilon}^{i / n}\left|g_{i, n}(s)\left(\sigma_{s-}-\sigma_{i / n-\varepsilon}\right)\right|^{\beta} d s\right]\right\}^{p / \beta} \\
& \leq C n^{-1+p(\alpha+1 / \beta)} \sum_{i=k}^{[n t]}\left\|v_{\sigma}(i / n, \varepsilon+1 / n)\right\|_{\beta}^{p}\left(\int_{i / n-\varepsilon}^{i / n}\left|g_{i, n}(s)\right|^{\beta} d s\right)^{p / \beta}
\end{aligned}
$$

By Lemma 4.7 and Lemma 4.8, we conclude that

$$
\lim _{\varepsilon \rightarrow 0} \limsup _{n \rightarrow \infty}\left(n^{-1+p(\alpha+1 / \beta)} \sum_{i=k}^{[n t]}\left\|A_{i}^{n, \varepsilon}\right\|_{p}^{p}\right)=0 .
$$


For $B_{i}^{n, \varepsilon}$ we apply Hölder's inequality with $p^{\prime}$ and $q^{\prime}$ satisfying $1 / p^{\prime}+1 / q^{\prime}=1$ and $p q^{\prime}<\beta$, which is possible due to our assumption $p<\beta$. This yields

$$
\begin{aligned}
& n^{-1+p(\alpha+1 / \beta)} \sum_{i=k}^{[n t]}\left\|B_{i}^{n, \varepsilon}\right\|_{p}^{p} \\
& \quad \leq n^{-1+p(\alpha+1 / \beta)} \sum_{i=k}^{[n t]}\left\|\left(\sigma_{i / n-\varepsilon}-\sigma_{(i-k) / n}\right)\right\|_{p p^{\prime}}^{p}\left\|\int_{i / n-\varepsilon}^{i / n} g_{i, n}(s) d L_{s}\right\|_{p q^{\prime}}^{p} \\
& \quad \leq C n^{-1} \sum_{i=k}^{[n t]}\left\|v_{\sigma}(i / n, \varepsilon+k / n)\right\|_{p p^{\prime}}^{p} .
\end{aligned}
$$

Here we have used that, as a consequence of Lemma 3.2 and Lemma 4.8, whenever $p q^{\prime}<\beta$ there exists a $C>0$ such that $\left\|n^{\alpha+1 / \beta} \int_{i / n-\varepsilon}^{i / n} g_{i, n}(s) d L_{s}\right\|_{p q^{\prime}}<C$ for all $n \in \mathbb{N}, i \in\{k, \ldots,[n t]\}$. Thus, by Lemma 4.7

$$
\lim _{\varepsilon \rightarrow 0} \limsup _{n \rightarrow \infty}\left(n^{-1+p(\alpha+1 / \beta)} \sum_{i=k}^{[n t]}\left\|B_{i}^{n, \varepsilon}\right\|_{p}^{p}\right)=0 .
$$

Moreover, by Lemma 3.2 and Lemma 4.8 it follows that for all $\varepsilon>0$

$$
\limsup _{n \rightarrow \infty}\left(n^{-1+p(\alpha+1 / \beta)} \sum_{i=k}^{[n t]}\left\|C_{i}^{n, \varepsilon}\right\|_{p}^{p}\right) \leq C \limsup _{n \rightarrow \infty}\left(n^{p(\alpha+1 / \beta-k)}\right)=0,
$$

which together with (4.16) and (4.17) completes the proof of (4.15).

By Minkowski's inequality for $p \geq 1$ and subadditivity for $p<1$, it is now sufficient to show that

$$
n^{-1+p(\alpha+1 / \beta)} \sum_{i=k}^{[n t]}\left|\sigma_{(i-1 / n)} \Delta_{i, k}^{n} G\right|^{p} \stackrel{\mathbb{P}}{\longrightarrow} m_{p} \int_{0}^{t}\left|\sigma_{s}\right|^{p} d s,
$$

in order to prove Theorem 1.1(ii).

Intuitively, replacing $\left|\Delta_{i, k}^{n} X\right|$ by $\left|\sigma_{(i-k) / n} \Delta_{i, k}^{n} G\right|$ corresponds to freezing the process $\left(\sigma_{t}\right)_{t \in \mathbb{R}}$ over blocks of length $1 / n$. For the proof of (4.18), we freeze $\sigma$ now over small blocks with block size $1 / l$ that does not depend on $n$. This will allow us to apply [13], Theorem 1.1(ii), on every block. Thereafter, (4.18) follows by letting $l \rightarrow \infty$. For $l>0$ we decompose

$$
\begin{aligned}
& n^{-1+p(\alpha+1 / \beta)} \sum_{i=k}^{[n t]}\left|\sigma_{(i-k) / n} \Delta_{i, k}^{n} G\right|^{p}-m_{p} \int_{0}^{t}\left|\sigma_{s}\right|^{p} d s \\
& =n^{-1+p(\alpha+1 / \beta)}\left(\sum_{i=k}^{[n t]}\left|\Delta_{i, k}^{n} G\right|^{p}\left(\left|\sigma_{(i-k) / n}\right|^{p}-\left|\sigma_{\left(j_{l, i}-1\right) / l}\right|^{p}\right)\right)
\end{aligned}
$$




$$
\begin{aligned}
& +\left(\sum_{j=1}^{[t]]+1}\left|\sigma_{(j-1) / l}\right|^{p}\left(n^{-1+p(\alpha+1 / \beta)} \sum_{i \in I_{l}(j)}\left|\Delta_{i, k}^{n} G\right|^{p}-m_{p} l^{-1}\right)\right) \\
& +\left(m_{p} l^{-1} \sum_{j=1}^{[t l]}\left|\sigma_{(j-1) / l}\right|^{p}-m_{p} \int_{0}^{t}\left|\sigma_{s}\right|^{p} d s\right) \\
& :=D_{n, l}+E_{n, l}+F_{l} .
\end{aligned}
$$

Here, $j_{l, i}$ denotes the index $j \in\{1, \ldots,[t l]+1\}$ such that $(i-k) / n \in((j-1) / l, j / l]$ and $I_{l}(j)$ is the set of indices $i$ such that $(i-k) / n \in((j-1) / l, j / l]$. We show that

$$
\lim _{l \rightarrow \infty} \limsup _{n \rightarrow \infty} \mathbb{P}\left(\left|D_{n, l}+E_{n, l}+F_{l}\right|>\varepsilon\right)=0
$$

for any $\varepsilon>0$. Note that $F_{l} \stackrel{\text { a.s. }}{\longrightarrow} 0$ as $l \rightarrow \infty$, since the Riemann integral of any càdlàg function exists. For every $l \in \mathbb{N}$, we have $\limsup _{n \rightarrow \infty} \mathbb{P}\left(\left|E_{n, l}\right|>\varepsilon\right)=0$ by [13], Theorem 1.1(ii). For $\lim _{l \rightarrow \infty} \lim \sup _{n \rightarrow \infty} \mathbb{P}\left(\left|D_{n, l}\right|>\varepsilon\right)=0$, we argue as follows. Choose some $p^{\prime}>1$ such that $p p^{\prime}<\beta$ and let $q^{\prime}$ be such that $1 / p^{\prime}+1 / q^{\prime}=1$. We find

$$
\begin{aligned}
\left\|D_{n, l}\right\|_{1} & =\left\|n^{-1+p(\alpha+1 / \beta)}\left(\sum_{i=k}^{[n t]}\left|\Delta_{i, k}^{n} G\right|^{p}\left(\left|\sigma_{(i-k) / n}\right|^{p}-\left|\sigma_{\left(j_{l, n, i}-1\right) / l}\right|^{p}\right)\right)\right\|_{1} \\
& \leq n^{-1} \sum_{i=k}^{[n t]}\left\|\left|n^{\alpha+1 / \beta} \Delta_{i, k}^{n} G\right|^{p}\right\|_{p^{\prime}}\left\|\left|\sigma_{(i-k) / n}\right|^{p}-\left|\sigma_{\left(j_{l, n, i}-1\right) / l}\right|^{p}\right\|_{q^{\prime}} \\
& \leq\left(n^{-1} \sum_{i=k}^{[n t]}\left\|n^{\alpha+1 / \beta} \Delta_{i, k}^{n} G\right\|_{p p^{\prime}}^{2 / p}\right)^{1 / 2}\left(n^{-1} \sum_{i=k}^{[n t]}\left\|\left|\sigma_{(i-k) / n}\right|^{p}-\left|\sigma_{\left(j_{l, n, i}-1\right) / l}\right|^{p}\right\|_{q^{\prime}}^{2}\right)^{1 / 2} .
\end{aligned}
$$

The first factor is bounded by Lemmas 3.2 and 4.8. For the second factor, we can apply Lemma 4.7 , since the process $\left(\left|\sigma_{t}\right|^{p}\right)_{t \in \mathbb{R}}$ is càdlàg and bounded on $[-\delta, \infty)$, and conclude that $\lim _{l \rightarrow \infty} \limsup _{n \rightarrow \infty}\left\|D_{n, l}\right\|_{1}=0$. This completes the proof of (4.18), and hence of Theorem 1.1(ii).

\subsection{Proof of Theorem 1.1(iii)}

For the proof of Theorem 1.1(iii), we show that under the conditions of the theorem the process $X$ admits a modification with $k$-times differentiable sample paths with $k$ th derivative $F$, as defined in the theorem. Then the result follows by an application of the following stochastic Fubini theorem. For a proof, we refer to [1], Theorem 3.1, where a similar Fubini theorem was shown for deterministic integrands. The generalisation towards predictable integrands is straightforward.

Lemma 4.9. Let $f: \mathbb{R} \times \mathbb{R} \times \Omega \rightarrow \mathbb{R}$ be a random field that is measurable with respect to the product $\sigma$-algebra $\mathcal{B}(\mathbb{R}) \otimes \Pi$, where $\Pi$ denotes the $\left(\mathcal{F}_{t}\right)_{t \in \mathbb{R}}$-predictable $\sigma$-algebra on $\mathbb{R} \times \Omega$. 
That is, $\Pi$ is the $\sigma$-algebra generated by all sets $A \times(s, t]$, where $s<t$ and $A \in \mathcal{F}_{s}$. Let $\left(L_{t}\right)_{t \in \mathbb{R}}$ be a symmetric Lévy process that has finite first moment. Assume that we have

$$
\mathbb{E}\left[\int_{\mathbb{R}}\|f(u, \cdot)\|_{1, L} d u\right]<\infty .
$$

Then, we obtain

$$
\int_{\mathbb{R}}\left(\int_{\mathbb{R}} f(u, s) d u\right) d L_{s}=\int_{\mathbb{R}}\left(\int_{\mathbb{R}} f(u, s) d L_{s}\right) d u \quad \text { almost surely, }
$$

and all the integrals are well-defined.

The following auxiliary result ensures that the conditions of this lemma are satisfied in our framework.

Lemma 4.10. Suppose that assumption (B1) holds. Let $q \in\{1, p\}$ which in particular implies $\alpha>k-1 /(\beta \vee q)$. If $q>1$ assume additionally that the jumps of the Lévy process $L$ are bounded in absolute value by 1 . For any $t>0$, the random field $f_{t}(u, s):=g^{(k)}(u-$ $s) \sigma_{s-} \mathbb{1}_{[0, t]}(u) \mathbb{1}_{(-\infty, u)}(s)$ satisfies

$$
\int_{0}^{t} \mathbb{E}\left[\left\|f_{t}(u, \cdot)\right\|_{q, L}^{q}\right] d u<\infty
$$

Proof. We decompose

$$
\begin{aligned}
& \int_{0}^{t} \mathbb{E}\left[\left\|f_{t}(u, \cdot)\right\|_{q, L}^{q}\right] d u \\
& \quad \leq C \int_{0}^{t} \mathbb{E}\left[\left\|f_{t}(u, \cdot) \mathbb{1}_{(-\delta, t]}\right\|_{q, L}^{q}\right] d u+C \int_{0}^{t} \mathbb{E}\left[\left\|f_{t}(u, \cdot) \mathbb{1}_{(-\infty,-\delta]}\right\|_{q, L}^{q}\right] d u \\
& \quad:=I_{1}+I_{2}
\end{aligned}
$$

and show that both summands are finite. For $I_{1}$ we use that $\sigma$ is bounded on $[-\delta, \infty)$. Thus, denoting $e_{t}(u, s)=g^{(k)}(u-s) \mathbb{1}_{[0, t]}(u) \mathbb{1}_{(-\delta, u)}(s)$, we obtain using (3.6)

$$
I_{1} \leq C \int_{0}^{t} \Phi_{q, L}\left(e_{t}(u, \cdot)\right)+\Phi_{q, L}^{\frac{q}{2}}\left(e_{t}(u, \cdot)\right) d u \leq C t\left(\Phi_{q, L}\left(e_{t}(t, \cdot)\right)+\Phi_{q, L}^{\frac{q}{2}}\left(e_{t}(t, \cdot)\right)\right),
$$

where in the second inequality we used $\left|e_{t}(u, s)\right| \leq\left|e_{t}(t, s+t-u)\right|$, and that $\Phi_{q, L}(f)$ is invariant under shifting the argument of the function $f$. For $I_{1}$ to be finite it is therefore sufficient to show that the following term is finite

$$
\int_{-\delta}^{t} \int_{\mathbb{R}}\left|g^{(k)}(t-s) x\right|^{2} \mathbb{1}_{\left\{\left|g^{(k)}(t-s) x\right| \leq 1\right\}}+\left|g^{(k)}(t-s) x\right|^{q} \mathbb{1}_{\left\{\left|g^{(k)}(t-s) x\right|>1\right\}} v(d x) d s:=J_{1}+J_{2} .
$$


We fix $\beta^{\prime} \in(\beta \vee 1,1 /(k-\alpha))$ and $q^{\prime} \in[q, 1 /(k-\alpha))$ such that the Lévy process satisfies $\mathbb{E}\left[\left|L_{1}\right|^{q^{\prime}}\right]<\infty$. Indeed, the former is possible by the conditions $\alpha>k-1 /(\beta \vee p)$ and $p \geq 1$ in Theorem 1.1(iii). The latter is possible for $q=1$ by the assumption $\theta>1$ in Theorem 1.1(iii), and for $q=p>1$ by the assumption of bounded jumps in the lemma. Recalling that $\left|g^{(k)}(t)\right| \leq$ $C|t|^{\alpha-k}$ for all $t \in(0, \delta)$, in order to show $J_{1}+J_{2}<\infty$, it is then sufficient to show

$$
J_{1}+J_{2} \leq C\left(1+\int_{-\delta}^{t}\left|g^{(k)}(t-s)\right|^{\beta^{\prime}} d s+\int_{-\delta}^{t}\left|g^{(k)}(t-s)\right|^{q^{\prime}} d s\right) .
$$

For $q=p>1$, this estimate follows easily from Lemma 4.5, where we use the assumption that $L$ has jumps bounded by 1 . For $q=1$ the estimate follows for $J_{1}$ by (3.4). For $J_{2}$ we obtain

$$
\begin{aligned}
J_{2} & \leq \int_{-\delta}^{t} \int_{-1}^{1}\left|g^{(k)}(t-s) x\right|^{\beta^{\prime}} \mathbb{1}_{\left\{\left|g^{(k)}(t-s) x\right|>1\right\}} v(d x) d s+2 \int_{-\delta}^{t}\left|g^{(k)}(t-s)\right|^{q^{\prime}} d s \int_{1}^{\infty}|x|^{q^{\prime}} v(d x) \\
& \leq C \int_{-\delta}^{t}\left|g^{(k)}(t-s)\right|^{\beta^{\prime}} \mathbb{1}_{\left\{\left|g^{(k)}(t-s)\right|>1\right\}}+\left|g^{(k)}(t-s)\right|^{q^{\prime}} d s,
\end{aligned}
$$

which concludes the proof of (4.19) and of $I_{1}<\infty$. For $I_{2}$ we use that $\left|g^{(k)}\right|$ is decreasing on $(\delta, \infty)$, which implies that $I_{2} \leq C t \mathbb{E}\left[\left\|f_{t}(0, \cdot) \mathbb{1}_{(-\infty,-\delta]}\right\|_{q, L}^{q}\right]$. By (3.6), the latter is finite if $\Phi_{q, L}^{1 \vee \frac{q}{2}}\left(f_{t}(0, \cdot) \mathbb{1}_{(-\infty,-\delta]}\right) \in L^{1}(\Omega)$. This follows easily from Assumption (B2) (recall that $\left.q \leq p\right)$ and (3.4).

With these preliminaries at hand, we can finally prove Theorem 1.1(iii). As remarked at the beginning of Section 4.2, it is sufficient to show convergence in probability for a fixed $t>0$ in order to obtain uniform convergence on compacts in probability. Therefore, the theorem is an immediate consequence of the following result and Lemma 4.3 in [13].

Lemma 4.11. Under the conditions of Theorem 1.1(iii), there is a process $\left(Z_{t}\right)_{t \geq 0}$ that satisfies almost surely $V(Z, p ; k)_{t}^{n}=V(X, p ; k)_{t}^{n}$ for all $n \in \mathbb{N}$ and $t \geq 0$, has almost surely $k$-times absolutely continuous sample paths and satisfies for Lebesgue almost all $t \geq 0$ that

$$
\frac{\partial^{k} Z_{t}}{(\partial t)^{k}}=\int_{-\infty}^{t} g^{(k)}(t-s) \sigma_{s-} d L_{s}:=F_{t},
$$

and $F \in L^{p}\left(\left[0, t_{0}\right]\right)$ for any $t_{0}>0$.

Proof. For ease of notation we only consider $k=1$. The general case follows by similar arguments. We let $a \in(0,1]$ and define the processes $\left(F_{u}^{\leq a}\right)_{u \in \mathbb{R}}$ and $\left(F_{u}^{>a}\right)_{u \in \mathbb{R}}$ by

$$
F_{u}^{\leq a}=\int_{-\infty}^{u} g^{\prime}(u-s) \sigma_{s-} d L_{s}^{\leq a}, \quad \text { and } \quad F_{u}^{>a}=\sum_{s \in(-\infty, u)} g^{\prime}(u-s) \sigma_{s-} \Delta L_{s} \mathbb{1}_{\left\{\left|\Delta L_{s}\right|>a\right\}},
$$

where the process $\left(L_{t}^{\leq a}\right)_{t \in \mathbb{R}}$ is the truncated Lévy process introduced in Section 4.1.2. We show that both processes $F_{u}^{\leq a}$ and $F_{u}^{>a}$ are well-defined and that they both admit a modification with 
sample paths in $L^{p}([0, t])$. Then, we define the process

$$
Z_{t}:=\int_{0}^{t}\left(F_{u}^{\leq a}+F_{u}^{>a}\right) d u
$$

and show that it satisfies the properties given in the lemma.

We begin by analysing $F_{u}^{\leq a}$. It is well-defined, since, as a consequence of Lemma 4.10, $f_{t_{0}}(u, s)=g^{\prime}(u-s) \sigma_{s-} \mathbb{1}_{\left[0, t_{0}\right]}(u) \mathbb{1}_{(-\infty, u)}(s)$ is integrable in $s$ with respect to $L^{\leq a}$ for Lebesgue almost all $u$. Applying Lemmas 3.1 and 4.10, we obtain $F^{\leq a} \in L^{p}([0, t])$, almost surely, since

$$
\mathbb{E}\left[\int_{0}^{t}\left|F_{u}^{\leq a}\right|^{p} d u\right] \leq C \int_{0}^{t} \mathbb{E}\left[\left\|f_{t}(u, \cdot)\right\|_{p, L \leq a}^{p}\right] d u<\infty .
$$

For the process $F_{u}^{>a}$ we make the decomposition

$$
\begin{aligned}
F_{u}^{>a} & =F_{u}^{>a, \leq-\delta}+F_{u}^{>a,>-\delta} \\
& =\sum_{s \in(-\infty,-\delta]} g^{\prime}(u-s) \sigma_{s-} \Delta L_{s} \mathbb{1}_{\left\{\left|\Delta L_{s}\right|>a\right\}}+\sum_{s \in(-\delta, u)} g^{\prime}(u-s) \sigma_{s-} \Delta L_{s} \mathbb{1}_{\left\{\left|\Delta L_{s}\right|>a\right\}} .
\end{aligned}
$$

We argue first that $F^{>a, \leq-\delta}$ is well-defined and in $L^{p}([0, t])$ almost surely. Applying Lemma 4.4, we obtain that

$$
\sum_{s \in(-\infty,-\delta]}\left|g^{\prime}(-s) \sigma_{s-} \Delta L_{s}\right| \mathbb{1}_{\left\{\left|\Delta L_{s}\right|>a\right\}}<\infty
$$

almost surely. Since $\left|g^{\prime}\right|$ is decreasing on $[\delta, \infty)$, this implies that $F^{>a, \leq-\delta}$ is well-defined and uniformly bounded in $u$. For $F_{u}^{>a,>-\delta}$, we use that $L$ has only finitely many jumps of size $>a$ on $[-\delta, t]$. Therefore, $F^{>a,>-\delta}$ is well-defined and we find a positive random variable $K<\infty$ such that

$$
\begin{aligned}
\int_{0}^{t}\left|F_{u}^{>a,>-\delta}\right|^{p} d u & \leq K \int_{0}^{t} \sum_{s \in(-\delta, u)}\left|g^{\prime}(u-s) \sigma_{s-\Delta} \Delta L_{s} \mathbb{1}_{\left\{\left|\Delta L_{s}\right|>a\right\}}\right|^{p} d u \\
& \leq K \sum_{s \in(-\delta, t)}\left|\sigma_{s-} \Delta L_{s} \mathbb{1}_{\left\{\left|\Delta L_{s}\right|>a\right\}}\right|^{p} \int_{0}^{t}\left|g^{\prime}(u-s)\right|^{p} d u
\end{aligned}
$$

which is finite since $\left|g^{\prime}(s)\right| \leq C s^{\alpha-1}$ for $s \in(0, \delta)$ and $(\alpha-1) p>-1$. All that remains to show is that $V(X, p ; 1)_{t}^{n}=V(Z, p ; 1)_{t}^{n}$ for all $n \in \mathbb{N}$ and all $t>0$ with probability 1 . For any $t>0$, it holds with probability 1 that

$$
X_{t}-X_{0}=\int_{\mathbb{R}}\left(\int_{\mathbb{R}} f_{t}(u, s) d u\right) d L_{s}=\int_{\mathbb{R}}\left(\int_{\mathbb{R}} f_{t}(u, s) d L_{s}\right) d u=Z_{t},
$$

where we have applied Lemmas 4.9 and 4.10. Consequently, it holds that $\mathbb{P}\left[X_{t}=Z_{t}+X_{0}\right.$ for all $\left.t \in \mathbb{Q}_{+}\right]=1$ which implies $V(X, p ; 1)_{t}^{n}=V(Z, p ; 1)_{t}^{n}$ for all $n \in \mathbb{N}$ and all $t>0$ almost surely. 


\section{Acknowledgments}

We are very grateful to three anonymous referees and the editor for their helpful remarks. Claudio Heinrich and Mark Podolskij acknowledge financial support from the project "Ambit fields: probabilistic properties and statistical inference" funded by Villum Fonden.

\section{References}

[1] Barndorff-Nielsen, O.E. and Basse-O'Connor, A. (2011). Quasi Ornstein-Uhlenbeck processes. Bernoulli 17 916-941. MR2817611

[2] Barndorff-Nielsen, O.E., Benth, F.E. and Veraart, A.E.D. (2013). Modelling energy spot prices by volatility modulated Lévy-driven Volterra processes. Bernoulli 19 803-845. MR3079297

[3] Barndorff-Nielsen, O.E., Corcuera, J.M. and Podolskij, M. (2009). Power variation for Gaussian processes with stationary increments. Stochastic Process. Appl. 119 1845-1865. MR2519347

[4] Barndorff-Nielsen, O.E., Corcuera, J.M. and Podolskij, M. (2011). Multipower variation for Brownian semistationary processes. Bernoulli 17 1159-1194. MR2854768

[5] Barndorff-Nielsen, O.E., Corcuera, J.M. and Podolskij, M. (2013). Limit theorems for functionals of higher order differences of Brownian semi-stationary processes. In Prokhorov and Contemporary Probability Theory (A.N. Shiryaev, S.R.S. Varadhan and E.L. Presman, eds.). Springer Proc. Math. Stat. 33 69-96. Heidelberg: Springer. MR3070467

[6] Barndorff-Nielsen, O.E., Corcuera, J.M., Podolskij, M. and Woerner, J.H.C. (2009). Bipower variation for Gaussian processes with stationary increments. J. Appl. Probab. 46 132-150. MR2508510

[7] Barndorff-Nielsen, O.E., Graversen, S.E., Jacod, J., Podolskij, M. and Shephard, N. (2006). A central limit theorem for realised power and bipower variations of continuous semimartingales. In From Stochastic Calculus to Mathematical Finance (Yu. Kabanov, R. Liptser and J. Stoyanov, eds.) 33-68. Berlin: Springer. MR2233534

[8] Barndorff-Nielsen, O.E., Jensen, E.B.V., Jónsdóttir, K.Y. and Schmiegel, J. (2007). Spatio-temporal modelling - with a view to biological growth. In Statistical Methods for Spatio-Temporal Systems (B. Finkenstädt, L. Held and V. Isham, eds.) 47-75. London: Chapman \& Hall/CRC.

[9] Barndorff-Nielsen, O.E., Pakkanen, M.S. and Schmiegel, J. (2014). Assessing relative volatility/intermittency/energy dissipation. Electron. J. Stat. 8 1996-2021. MR3273617

[10] Barndorff-Nielsen, O.E. and Schmiegel, J. (2007). Ambit processes: With applications to turbulence and tumour growth. In Stochastic Analysis and Applications. Abel Symp. 2 93-124. Berlin: Springer. MR2397785

[11] Barndorff-Nielsen, O.E. and Schmiegel, J. (2008). Time change, volatility, and turbulence. In Mathematical Control Theory and Finance (A. Sarychev, A. Shiryaev, M. Guerra and M.d.R. Grossinho, eds.) 29-53. Berlin: Springer. MR2484103

[12] Barndorff-Nielsen, O.E. and Schmiegel, J. (2009). Brownian semistationary processes and volatility/intermittency. In Advanced Financial Modelling. Radon Ser. Comput. Appl. Math. 8 1-25. Berlin: Walter de Gruyter. MR2648456

[13] Basse-O'Connor, A., Lachièze-Rey, R. and Podolskij, M. (2016). Power variation for a class of stationary increments Lévy driven moving averages. Ann. Probab. To appear.

[14] Basse-O'Connor, A. and Podolskij, M. (2017). On critical cases in limit theory for stationary increments Lévy driven moving averages. Stochastics 89 360-383.

[15] Benassi, A., Cohen, S. and Istas, J. (2004). On roughness indices for fractional fields. Bernoulli 10 357-373. MR2046778 
[16] Billingsley, P. (1999). Convergence of Probability Measures, 2nd ed. Wiley Series in Probability and Statistics: Probability and Statistics. New York: Wiley. MR1700749

[17] Chronopoulou, A., Viens, F.G. and Tudor, C.A. (2009). Variations and Hurst index estimation for a Rosenblatt process using longer filters. Electron. J. Stat. 3 1393-1435. MR2578831

[18] Coeurjolly, J.-F. (2001). Estimating the parameters of a fractional Brownian motion by discrete variations of its sample paths. Stat. Inference Stoch. Process. 4 199-227. MR1856174

[19] Dang, T.T.N. and Istas, J. (2015). Estimation of the Hurst and the stability indices of a H-self-similar stable process. Working paper. Available at arXiv:1506.05593.

[20] Gärtner, K. and Podolskij, M. (2015). On non-standard limits of Brownian semi-stationary processes. Stochastic Process. Appl. 125 653-677. MR3293298

[21] Grahovac, D., Leonenko, N.N. and Taqqu, M.S. (2015). Scaling properties of the empirical structure function of linear fractional stable motion and estimation of its parameters. J. Stat. Phys. 158 105-119. MR3296276

[22] Guyon, X. and León, J. (1989). Convergence en loi des $H$-variations d'un processus gaussien stationnaire sur R. Ann. Inst. Henri Poincaré Probab. Stat. 25 265-282. MR1023952

[23] Jacod, J. (2008). Asymptotic properties of realized power variations and related functionals of semimartingales. Stochastic Process. Appl. 118 517-559. MR2394762

[24] Jacod, J. and Protter, P. (2012). Discretization of Processes. Stochastic Modelling and Applied Probability 67. Heidelberg: Springer. MR2859096

[25] Jacod, J. and Shiryaev, A.N. (2003). Limit Theorems for Stochastic Processes, 2nd ed. Berlin: Springer. MR1943877

[26] Kwapień, S. and Woyczyński, W.A. (1992). Random Series and Stochastic Integrals: Single and Multiple. Probability and Its Applications. Boston, MA: Birkhäuser. MR1167198

[27] Musielak, J. (1983). Orlicz Spaces and Modular Spaces. Lecture Notes in Math. 1034. Berlin: Springer. MR0724434

[28] Nourdin, I. and Réveillac, A. (2009). Asymptotic behavior of weighted quadratic variations of fractional Brownian motion: The critical case $H=1 / 4$. Ann. Probab. 37 2200-2230. MR2573556

[29] Podolskij, M. and Vetter, M. (2010). Understanding limit theorems for semimartingales: A short survey. Stat. Neerl. 64 329-351. MR2683464

[30] Rajput, B.S. and Rosiński, J. (1989). Spectral representations of infinitely divisible processes. Probab. Theory Related Fields 82 451-487. MR1001524

[31] Rosiński, J. and Woyczyński, W.A. (1986). On Itô stochastic integration with respect to $p$-stable motion: Inner clock, integrability of sample paths, double and multiple integrals. Ann. Probab. 14 271-286. MR0815970

[32] Sato, K. (1999). Lévy Processes and Infinitely Divisible Distributions. Cambridge Studies in Advanced Mathematics 68. Cambridge: Cambridge Univ. Press. MR1739520

[33] Skorohod, A.V. (1956). Limit theorems for stochastic processes. Theory Probab. Appl. 1 261-190.

[34] Tudor, C.A. and Viens, F.G. (2009). Variations and estimators for self-similarity parameters via Malliavin calculus. Ann. Probab. 37 2093-2134. MR2573552

[35] Whitt, W. (2002). Stochastic-Process Limits. New York: Springer. MR1876437

Received April 2016 and revised February 2017 